\section{A New Comprehensive Catalog of the Human Virome Reveals Hidden Associations with Chronic Diseases}

\author{
Michael J. Tisza and Christopher B. Buck*
}

\title{
Abstract
}

While there have been remarkable strides in microbiome research, the viral component of the microbiome has generally presented a more challenging target than the bacteriome. This is despite the fact that many thousands of shotgun sequencing runs from human metagenomic samples exist in public databases and all of them encompass large amounts of viral sequences. The lack of a comprehensive database for human-associated viruses, along with inadequate 7 methods for high-throughput identification of highly divergent viruses in metagenomic data, 8 has historically stymied efforts to characterize virus sequences in a comprehensive way. In this 9 study, a new high-specificity and high-sensitivity bioinformatic tool, Cenote-Taker 2, was applied to thousands of human metagenome datasets, uncovering over 50,000 unique virus 21 operational taxonomic units. Publicly available case-control studies were re-analyzed, and over 221,700 strong virus-disease associations were found. 
Introduction

The human virome is the sum total of all viruses that are intimately associated with people. This includes viruses that directly infect human cells ${ }^{1}$ but mostly consists of viruses infecting resident bacteria, i.e. phages ${ }^{2}$. While the large majority of microbiome studies have focused on the bacteriome, revealing numerous important functions for bacteria in human physiology ${ }^{3}$, information about the human virome has lagged. However, a number of recent studies have begun making inroads into characterizing the virome ${ }^{4-12}$.

Just as human-tropic viruses can have dramatic effects on people, phages are able to dramatically alter bacterial physiology and regulate host population size. A variety of evolutionary dynamics can be at play in the phage/bacterium arena, including Red Queen ${ }^{10}$, arms-race $^{13}$, and piggyback-the-winner ${ }^{14}$ relationships, to name just a few. In the gut, many phages enter a lysogenic or latent state and are retained as integrated or episomal prophages within the host bacterium ${ }^{15}$. In some instances, the prophage can buttress host fitness (at least temporarily) rather than destroying the host cell. Prophages often contain genes that can dramatically alter the phenotype of the bacteria, such as toxins ${ }^{16}$, virulence factors ${ }^{17}$, antibiotic resistance genes ${ }^{18}$, photosystem components ${ }^{19}$, other auxiliary metabolic genes ${ }^{20}$, and CRISPRCas systems ${ }^{21}$, along with countless genes of unknown function. Experimental evidence has shown that bacteria infected with particular phages - i.e. "virocells" - are physiologically distinct from cognate bacteria that lack those particular phages ${ }^{20}$.

There have been a few documented cases where phages have been shown to be involved in increased bacterial virulence ${ }^{16}$ or resistance to antibiotics ${ }^{22}$, demonstrating complex roles for phages in human health. In addition, several studies have conducted massively parallel sequencing on virus-enriched samples of human stool, finding differential abundance of some phages in disease conditions ${ }^{5,23-25}$. A major issue encountered by these studies is that there is not yet a comprehensive database of annotated virus genome sequences, and de novo prediction of virus sequences from metagenomic assemblies remains a daunting challenge ${ }^{2}$. In one study, only $31 \%$ of the assembled sequence data in virion-enriched virome surveys could be identified as recognizably viral ${ }^{26}$. Impressively, another study of twelve individuals was able to recruit over $80 \%$ of reads from virus-enriched samples to putative virus contigs ${ }^{10}$. Still, most of the potential viral contigs were unclassifiable sequences, and a large majority of contigs appeared to represent subgenomic fragments under $10 \mathrm{~kb}$.

The current study sought to overcome the traditional challenges of sparse viral databases and poor detection of highly divergent viral sequences by using Cenote-Taker 2, a new virus discovery and annotation tool ${ }^{27}$. The pipeline was applied to sequencing data from nearly 6,000 human metagenome samples. Strict criteria identified over 180,000 viral contigs representing 52,570 specific taxa. In most cases, $75-99 \%$ of reads from virus-enriched stool datasets could be back-aligned to the Cenote-Taker 2-compiled Human Virome Database. Furthermore, the curated database allowed read-alignment-based abundance profiling of the 
62 virome in human metagenomic datasets, enabling the re-analysis of a panel of existing case-

63 control studies. The re-analysis revealed previously undetected associations between chronic

64 diseases and the abundance of 1,789 specific virus taxa.

\section{Results}

68 Read data was downloaded from NCBI's Sequence Read Archive (SRA), including data from the Human Microbiome Project ${ }^{28}$ and several other studies (25 Bioprojects in total) involving deep

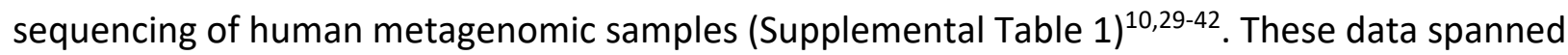
multiple body sites, including gut (stool), mouth, skin, and vagina. A subset of the projects performed enrichment for viral sequences ${ }^{43-46}$. Almost all of the projects pursued DNA sequencing, but a small number of metatranscriptomic (i.e., ribosome-depleted total RNA) samples were analyzed as well ${ }^{47}$. Read data were binned and assembled by Biosample rather than by individual run to combine read sets from the same individual. A total of 5,996 Biosamples were analyzed.

Cenote-Taker $2^{27}$ was used to check contigs for two common end-features of complete viral genomes: terminal direct repeats (suggesting a circular or LTR-bounded viral genome) or inverted terminal repeats (ITRs). Sequences with direct terminal repeats were arbitrarily assumed to represent circular DNA genomes. Circularized sequences $>1,500$ nucleotides (nt) with at least one viral hallmark gene and ITR-containing contigs $>4,000 \mathrm{nt}$ with at least one viral hallmark gene were designated as putative viruses. Linear (no discernable end features) contigs $>12,000$ nt with two or more viral hallmark genes were also kept as putative viruses. Since phages are often integrated into bacterial chromosomes, each linear contig was pruned with the Cenote-Taker 2 prophage pruning module to remove flanking host sequences. The analysis resulted in over 180,000 high-quality putative viral sequences. The sequences were classified into operational taxonomic units (OTUs) by clustering at $<0.05$ intersequence distance (a proxy for $>95 \%$ average nucleotide identity) using Mash $^{48}$ (see Methods). A final database of 52,570 sequences representing nonredundant virus OTUs was generated, and this database will herein be referred to as the Cenote Human Virome Database (CHVD, download available at https://zenodo.org/record/4069377).

8,625 virus OTUs consisted of contigs with terminal direct repeats and 113 were ITRbounded. An additional 2,070 virus OTUs were likely complete proviruses because they were flanked on both sides by host sequences. Since it can be difficult to detect many kinds of viral genome ends using short read assemblies the absence of terminal repeats or flanking chromosomal regions does not necessarily imply that a given contig represents an incomplete viral genome (or genome segment) ${ }^{49}$. It is thus uncertain what proportion of the remaining 41,762 contigs in the CHVD represent complete genomes, as opposed to subgenomic 99 fragments. 
Although it is often challenging to obtain very long virus contigs from de novo assemblies, 133 virus OTUs over 200 kilobases $(\mathrm{kb})$ were detected in the survey, with the largest being Siphoviridae species ctpHQ1, at $501 \mathrm{~kb}$. A total of 38 family- or order-level taxa were observed, and 2,568 sequences could not be classified by Cenote-Taker 2 (Supplemental Fig. 1). It is important to note that virus taxonomy, especially taxonomy of dsDNA phages, is currently in flux ${ }^{50-53}$, and these taxonomic statistics will likely change as taxonomic groupings are revised. The vast majority of classified sequences represent dsDNA tailed phages in the order Caudovirales (Including Siphoviridae, Podoviridae, Myoviridae, Ackmannviridae, Herelleviridae, and CrAss-like viruses). Relatively small numbers of known human-tropic viruses were uncovered, including members of families Adenoviridae, Anelloviridae, Circoviridae, Herpesviridae, Caliciviridae (Human norovirus), Papillomaviridae, and Polyomaviridae. Most of the human-tropic viruses mapped to previously reported virus species, but 16 previously undiscovered anelloviruses were detected (download available from: https://zenodo.org/record/4069377). Supplemental Table 2 contains information on each virus, including OTU, hallmark genes, CRISPR hits (see below), and statistical information.

Figure 1 presents a graphical summary of observed virus taxa. One taxon, designated "Phyco-like_phage", is represented by 51 contigs. This is an interesting group of sequences defined by Cenote-Taker 2 as Phycodnaviridae due to similarity of the terminase/packaging gene of these viruses to a gene encoded by eukaryotic phycodnaviruses ( $30 \%$ AA similarity). However, most of the inferred virion structural genes that co-occupy these contigs are distantly similar to those of crAss-like phages, not phycodnaviruses, suggesting that they represent phages. This, and the fact that most of the 2,570 "Unclassified" viral sequences have virion hallmark genes corresponding to dsDNA phage models (Supplemental Fig. 1) (Cytoscape network file available from: https://zenodo.org/record/4069377), supports the idea that substantial phage diversity remains unclassified and undescribed.

To evaluate the degree to which the observed CHVD virus OTUs are already represented in public databases, Mash was used to measure roughly intraspecies-level nucleotide sequence similarity to 23,386 genomes from annotated virus species found in GenBank. With a Mash distance threshold of $<0.05,366 / 52,570$ (0.7\%) Cenote Human Virome Database viruses had at least one strict cognate sequence in GenBank (Supplemental Table 3). If the Mash distance threshold is relaxed to $<0.1,2,658 / 52,570$ (5.1\%) Cenote Human Virome Database viruses have a GenBank cognate (Supplemental Table 3). different virus discovery methods. The Cenote Human Virome Database presented in this manuscript contains sequences from multiple human body sites, so comparisons are not perfect. However, we note that CHVD has $58 \%$ more contigs $(52,570$ vs 33,242$)$ and $181 \%$ more sequence information (1.674 gigabases vs 0.596 gigabases) than GVD. The same Mash distance analysis was applied to compare the two datasets. With a strict threshold, $6,832(13.0 \%)$ virus 
138 sequences from this study had a cognate in the Gut Virome Database. At the looser threshold, 13920,990 (40.0\%) sequences had cognates in both databases (Supplemental Table 4).

140 An important element of the Cenote Taker 2 pipeline is that it provides output files

141 suitable for submission to GenBank. At the time of manuscript submission, the process of

142 depositing all CHVD OTU genome maps into GenBank is ongoing.

143 
bioRxiv preprint doi: https://doi.org/10.1101/2020 11.01.363820; this version posted November 1,2020. The copyright holder for this preprint (which was not certified by peer review) is the author/funder. This article is a US Government work. It is not subject to copyright under 17 USC 105 and is also made available for use under a CCO license.

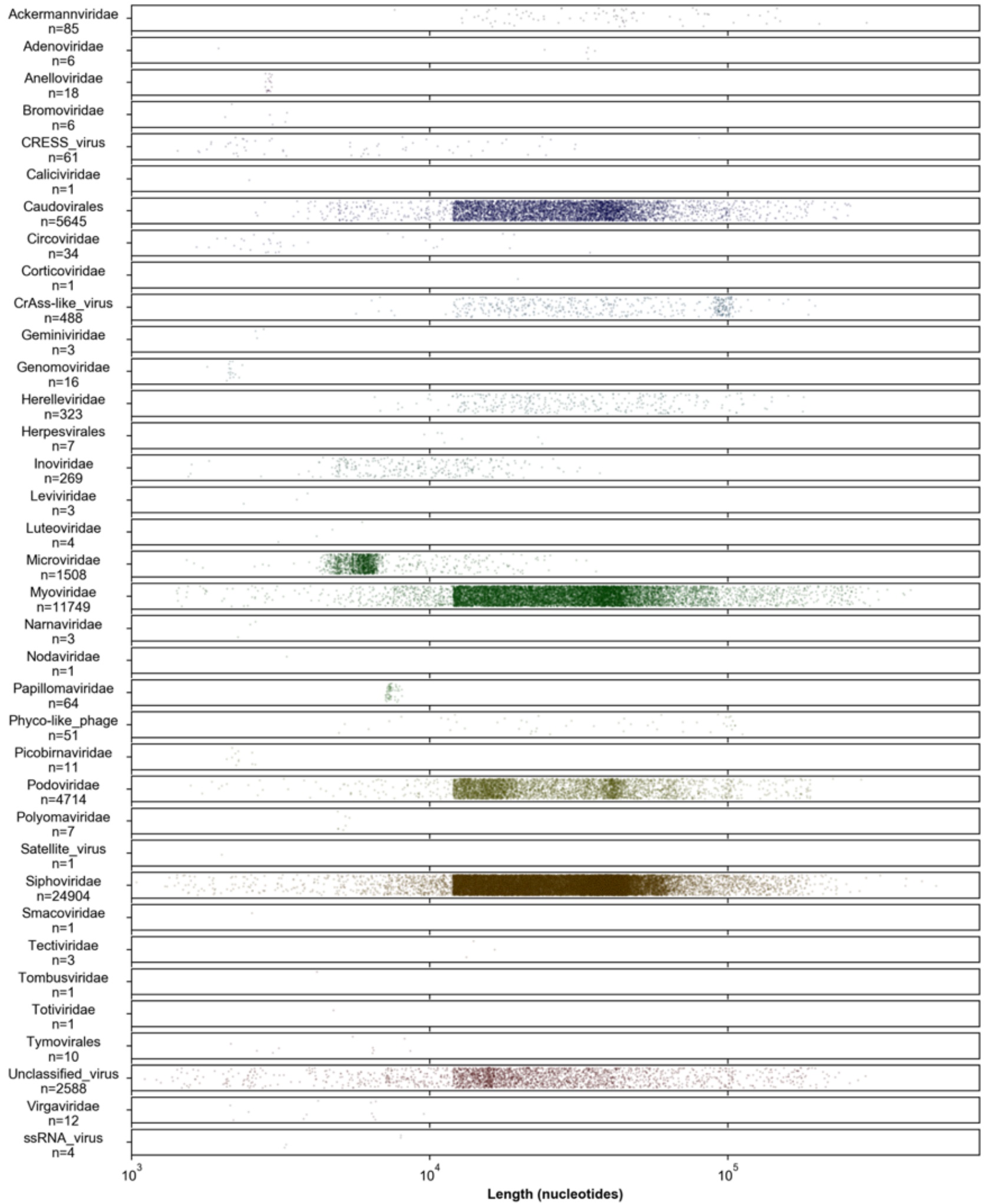

Figure 1: Summary of virus contig taxonomy and length

146 Each classified contig is represented as dot, with the $\mathrm{x}$-axis position representing contig length. 
bioRxiv preprint doi: https://doi.org/10.1101/202011.01.363820; this version posted November 1, 2020. The copyright holder for this preprint

(which was not certified by peer review) is the author/funder. This article is a US Government work. It is not subject to copyright under 17 USC 105 and is also made available for use under a CCO license.

148 bounded by terminal repeats, or the result of pruning of inferred bacterial chromosomal

149 sequences. Larger (> 3kb) CRESS virus OTUs consist of contigs in a previously reported taxon

150 that combines CRESS-like replication genes with inovirus-like virion genes ${ }^{54,55}$.

151 
bioRxiv preprint doi: https://doi.org/10.1101/202011.01.363820; this version posted November 1, 2020. The copyright holder for this preprint (which was not certified by peer review) is the author/funder. This article is a US Government work. It is not subject to copyright under 17 USC 105 and is also made available for use under a CCO license.
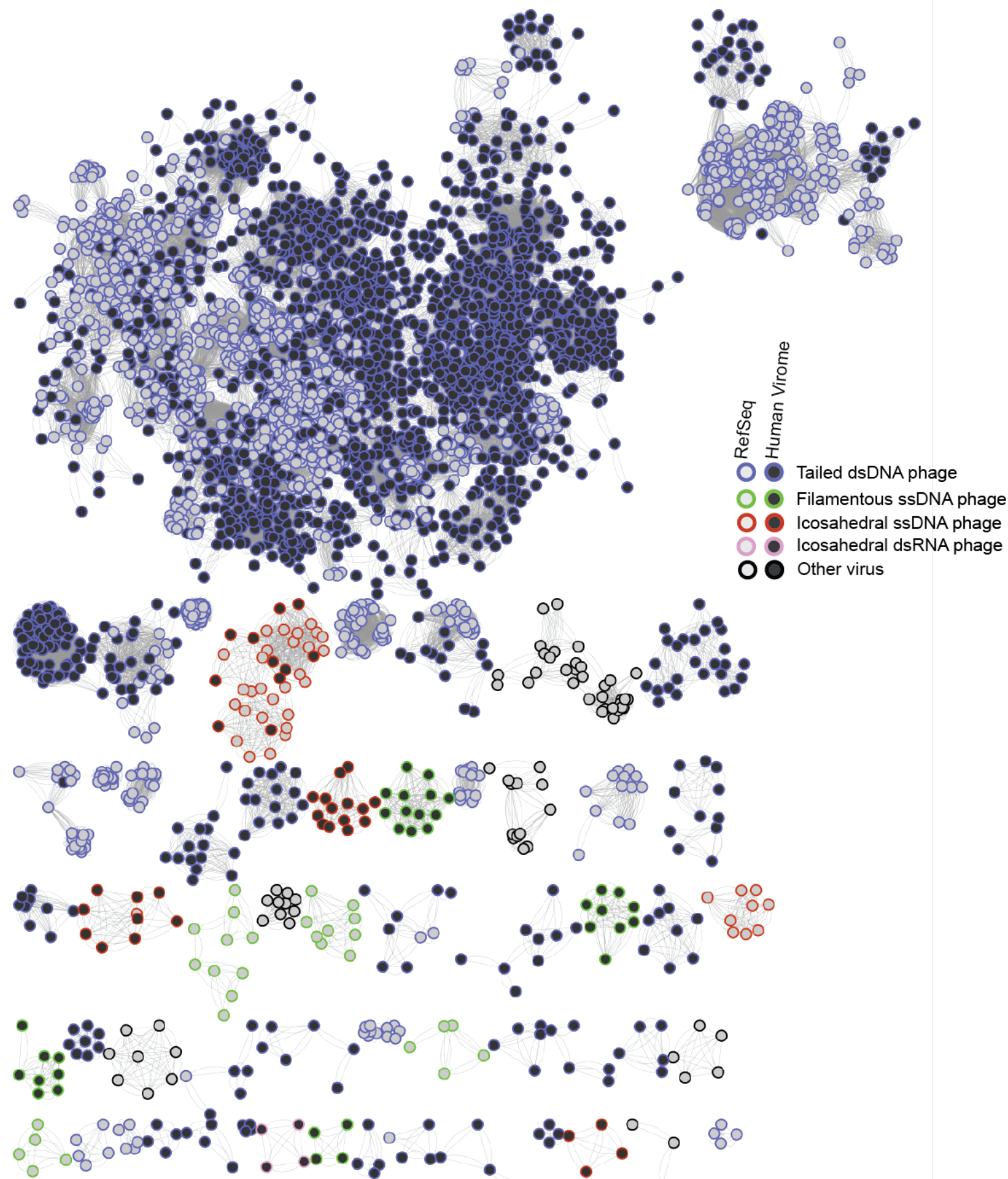

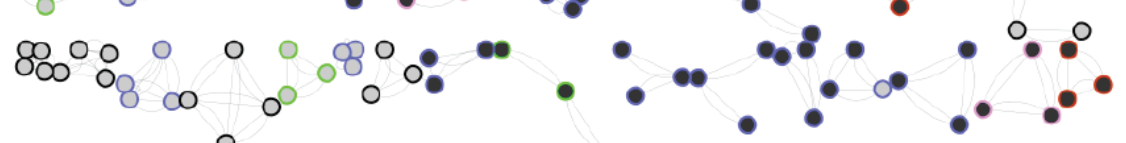
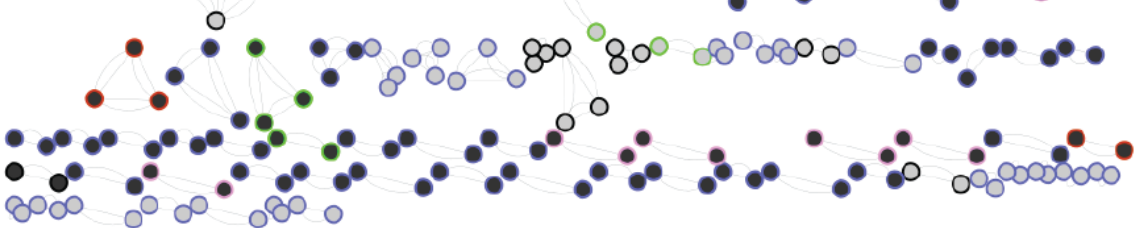
154 Vcontact $2^{52}$ was used to make a network of RefSeq bacteriophage genomes and CHVD contigs 155 deemed "unclassified viruses." Vcontact2 is more sensitive than the taxonomy module of

156 Cenote-Taker 2, as Vcontact2 compares all genes encoded on each contig and Cenote-Taker 2

157 uses only a single hallmark gene for comparison to its taxonomy database. Edges are drawn

158 between nodes if some proportion of their genes share protein sequence similarity. Only

159 clusters of two or more nodes are displayed. Each sequence cluster was given a feature label,

160 such as "filamentous ssDNA phage," based on manual inspection of the virion hallmark gene

161 calls made by Cenote-Taker 2 (see Supplemental Table 2) for one or more sequences in the

162 cluster. 
164 The large majority of reads from well-enriched virome preparations are identifiable

It is unclear how much of the human virome is cataloged by CHVD. One way to address

167 determine what fraction of reads in the dataset are identifiable. Reads from 983 human stool

168 samples (representing 11 different studies) that were physically enriched for virions and

169 subjected to nuclease digestion to remove non-encapsidated nucleic acids were mapped

170 against the CHVD (Fig. 2A) (Supplemental Table 5). For most samples with high virus enrichment

171 scores, over $75 \%$ of reads were mappable. The result indicates that the database accounts for a

172 large majority of the human virome, at least at a sequence abundance level.

173 Though well-enriched viromic data were not as available for other body sites, roughly

$17475 \%$ of reads were classifiable in well-enriched oral samples enriched for virus DNA (Fig. 2B)

175 (Supplemental Table 5). 
bioRxiv preprint doi: $h t t p s: / / d o i$ org/10.1101/202011.01363820; this version posted November 1 2020. The copyright holder for this preprint (which was not certified by peer review) is the author/funder. This article is a US Government work. It is not subject to copyright under 17 USC 105 and is also made available for use under a CCO license.

177

A

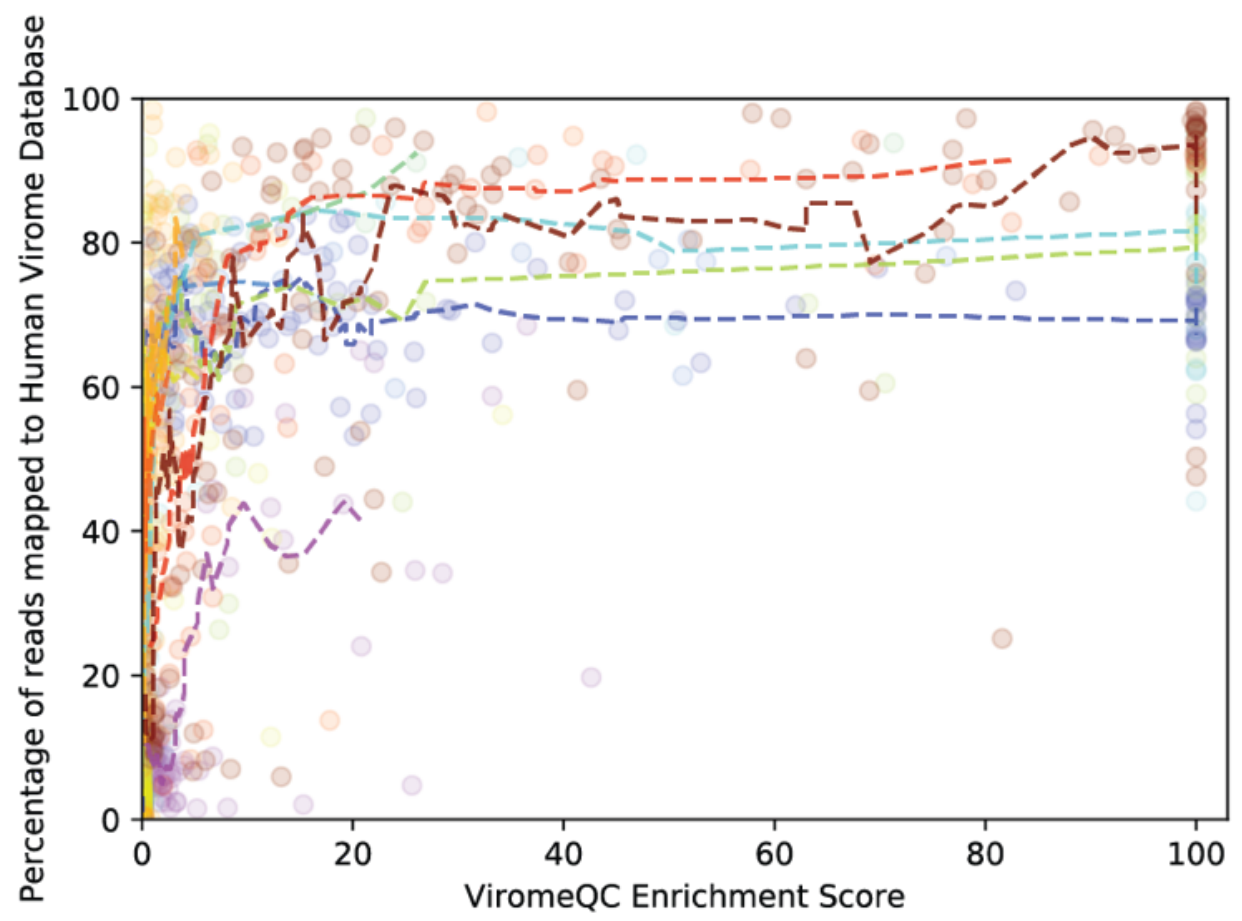

- - PRJEB9524

- - PRJEB9818

- - PRJNA196801

- - PRJNA284162

- - PRJNA308867

- - PRJNA385126

- - PRJNA387903

- - PRJNA389927

- - PRJNA491626 *

-- PRJNA498332*

- - PRJNA545408 *

B

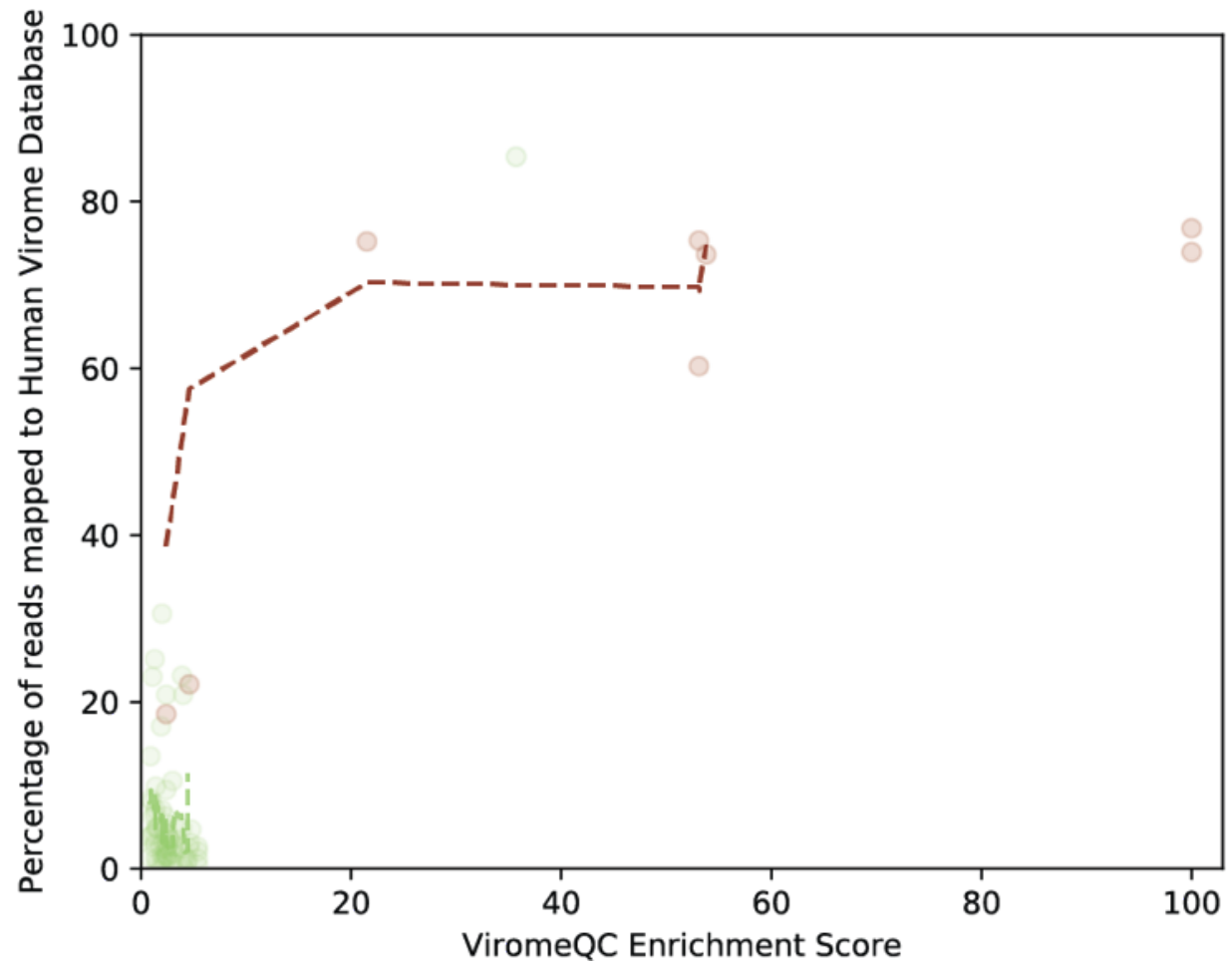

- - PRJEB22882

- - PRJNA413824 
179 Figure 2: Alignment of reads from "virion-enriched preparations" to the Cenote Human Virome

180 Database

181 Data from virion-enriched samples are plotted. To measure the degree to which enrichment for

182 viral sequences was achieved, a ViromeQC Enrichment Score ${ }^{26}$ was calculated for each sample

183 (x-axis). The enrichment score is essentially the inverse abundance of known bacterial single-

184 copy marker genes. Dotted lines are moving averages of samples from the same study.

185 Asterisks indicate Bioprojects with data used in the creation of the Cenote Human Virome

186 Database. Panel A shows gut (stool) virome samples, Panel B shows oral (saliva) viromes. A

187 modified database where sequences were clustered at 99\% identity instead of 95\% identity was

188 used for the index to better capture microdiversity and metaviromic islands ${ }^{56}$ (e.g. intraspecific

189 structural variations consisting of insertions/deletions of gene cassettes, see Methods). 
CRISPR spacer analysis reveals candidate hosts for most phages as well as phage-phage competition networks

Many bacteria encode CRISPR-Cas systems, which contain CRISPR spacer arrays of short ( $32 \mathrm{nt}$ ) sequences copied from and used against invading mobile genetic elements, especially phages ${ }^{57}$. Matching bacterial CRISPR spacers to phage genomes is one way to determine if a bacterial lineage has previously been exposed to a particular phage. Advances in cataloging of CRISPR spacers from bacterial genomes and optimization of phage/host matching pipelines allowed the association of most of the phages discovered in this project to bacterial hosts (http://crispr.genome.ulaval.ca/). Specifically, 36,057 of the 52,570 virus sequences had at least one CRISPR spacer match from a known bacterium or multiple bacteria, with 416,483 total unique spacers matched to Cenote Human Virome Database sequences (Supplemental Table 2). CRISPR spacer density varied dramatically among different bacterial taxa (Fig. 3). For example, members of genus Bifidobacterium were confirmed to have relatively large and diverse CRISPR spacer libraries ${ }^{58}$, while Clostridium, Porphyromonas, and Leptotrichia typically encoded one or 205 only a handful of spacers per phage.

CrAss-like viruses seem to be the target of relatively few spacers per virus OTU, despite the fact many representative contigs in CHVD are $\sim 100 \mathrm{~kb}$. In contrast, ssDNA phages of family

208 Microviridae, despite their small size, seem to be frequently targeted by many unique spacers 209 in Bacteroides and Parabacteroides CRISPR systems but not CRISPR systems in other bacterial 210 taxa.

211 Phages themselves can encode CRISPR arrays and some phages have intact and 212 functional CRISPR-Cas systems ${ }^{21,59}$. These CRISPR components can target host defenses as well 213 as other phages competing for the same host ${ }^{60}$. Among phage sequences in the Cenote Human 214 Virome Database, 2,091 CRISPR spacers were detected in arrays from the genomes of 223 215 phages. Of these, 875 spacers targeted a total of 2,491 other phages, suggesting complex 216 phage-phage competition networks in human metagenomes (Fig. 4) (download cytoscape file 217 from: https://zenodo.org/record/4069377). 

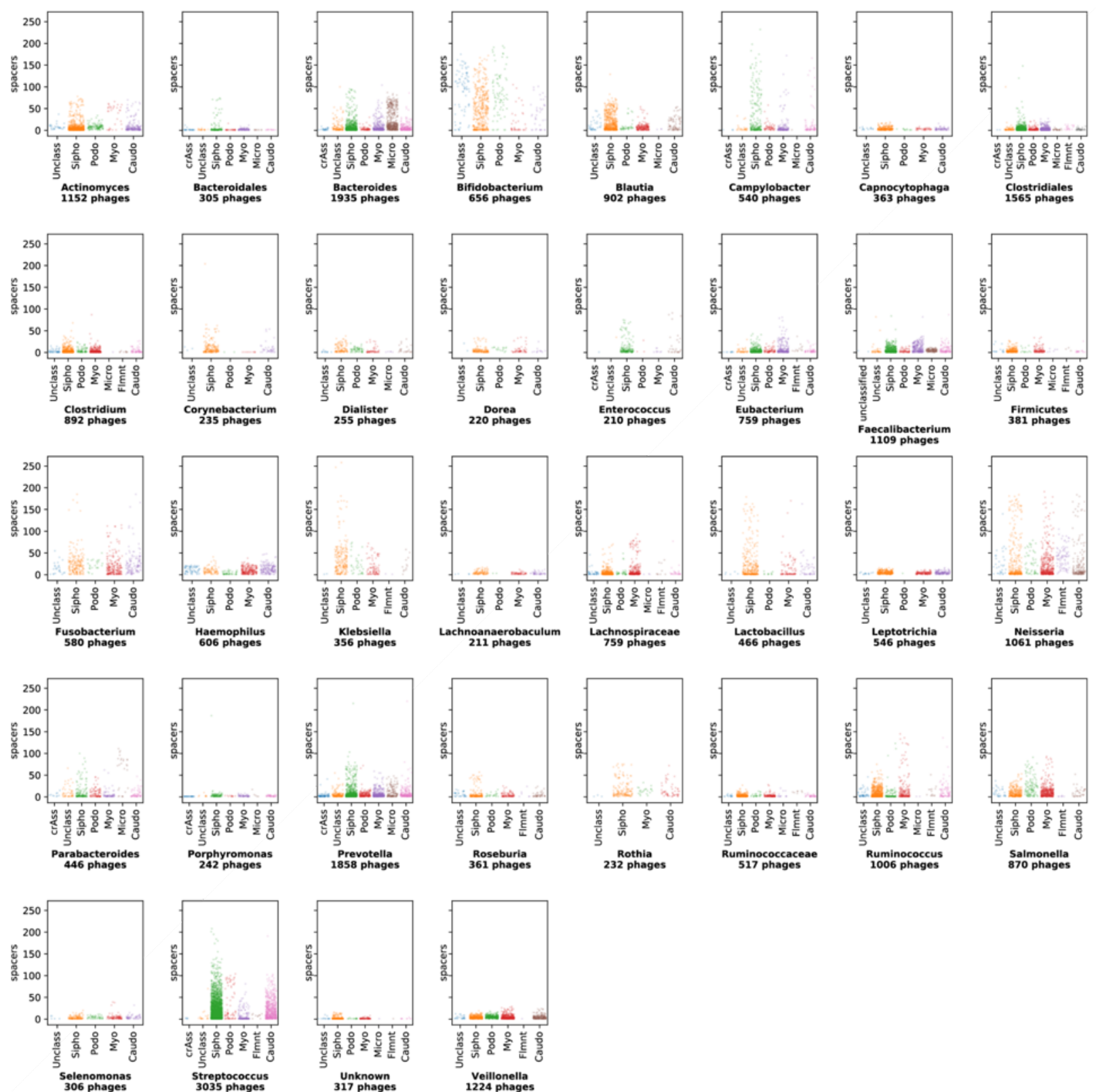

220

Figure 3: Summary of CRISPR spacer matches to bacterial taxa

221 Each plot represents data from a different bacterial genus (or higher taxon when genus is not

222 clearly defined, see methods) with CRISPR spacer matches to 200 or more CHVD OTUs. Y-axis

223 values represent the number of unique bacterial CRISPR spacer hits for each virus OTU. Myo =

224 Myoviridae, Sipho $=$ Siphoviridae, crAss $=$ CrAss-like viruses, Caudo $=$ Other Caudovirales, Micro

$225=$ Microviridae, FImnt = Inoviridae and other filamentous phages or CRESS viruses, Unclass =

226 unclassified viruses 


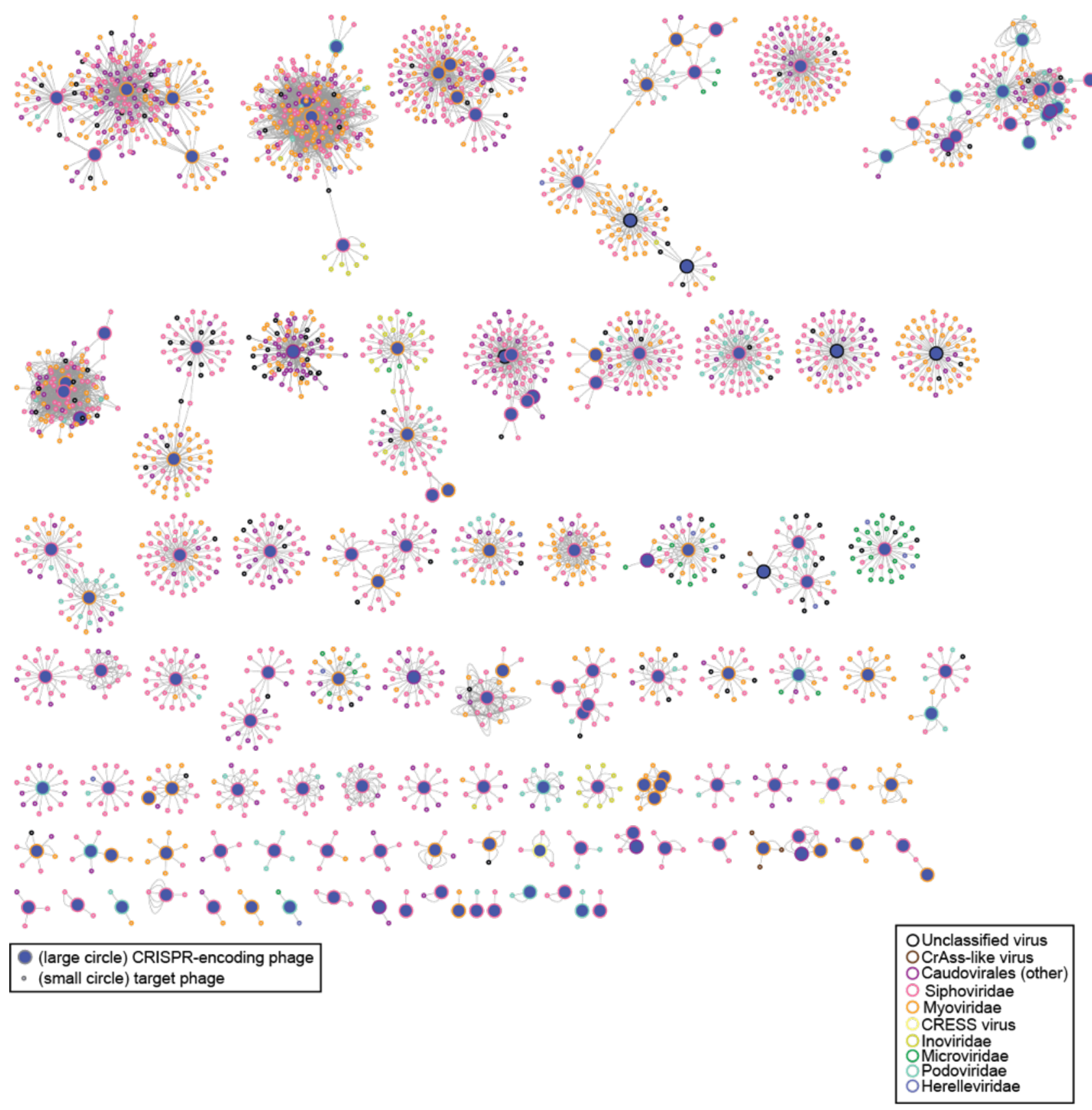

Figure 4: Phage-encoded CRISPR spacers reveal phage interaction networks in the human

230 virome

231 Network diagram of phage-phage interaction landscape based on CRISPR spacer matches. Each

232 line represents a match of a particular spacer sequence to its target phage. 
The most commonly abundant viruses on several body sites

With this library of viruses and the large sampling effort from the Human Microbiome Project ${ }^{28,61}$, the question of "which viruses are the most commonly abundant" for a given body site can be answered more confidently than was previously possible. It should be noted that the Human Microbiome Project data was collected from healthy Americans between 18 and 40 years of age and the conclusions here may not be generalizable to other populations.

It is often challenging to precisely determine the border between an integrated prophage and host chromosomal sequences without experimental validation. Our preliminary analyses revealed that inclusion of even a few hundred nucleotides of flanking host sequence in a viral OTU can greatly distort abundance measurements because of inadvertent measurement of uninfected host bacterial sequences. We therefore used Cenote-Taker 2 to perform a more stringent analysis in which contigs were trimmed from the first recognized virus hallmark gene through the last virus hallmark gene. We refer to these more stringent units as "virus cores" (download from: https://zenodo.org/record/4069377)

Data were downloaded from SRA and analyzed for hundreds of patients at six body sites (anterior nares, buccal mucosa, posterior fornix, tongue dorsum, supragingival plaque, and gut (stool)). Reads were then aligned to the more stringent virus cores database. As a proxy for the relative abundance of a given virus OTU, the average number of reads per kilobase of virus genome per million reads in the parent dataset (RPKM) was calculated for each sequence, (Fig 5, Supplemental Figs 2-4). Virus prevalence was determined as proportion of samples with $>0.1$ RPKM. The most commonly abundant virus OTUs were calculated as (mean RPKM $\times$ prevalence). The right panel of Fig 5 shows the inferred host for each of the top 50 most commonly abundant virus OTUs based on CRISPR spacer target information. A majority of the most of the commonly abundant viruses appear to infect members of the common bacterial phylum Bacteroidetes, which is generally abundant in the human gut.

The data suggest an interesting bifurcation in prevalence of virus OTUs with high abundance (RPKM). Although some virus OTUs, such as Podoviridae sp. ctBGm1 and Myoviridae sp. ctiOo1, are present in nearly all samples and have an average abundance of $>10$ RPKM, perhaps representing prophage of ubiquitous bacterial lineages. Others, including all displayed CrAss-like viruses and Caudovirales sp. ctdGD4, are absent or low-abundance in most samples but highly abundant in a minority of samples. The latter group could either represent viruses that periodically undergo large replicative bursts, or viruses that constitutively dominate the virome in certain individuals but not others. 


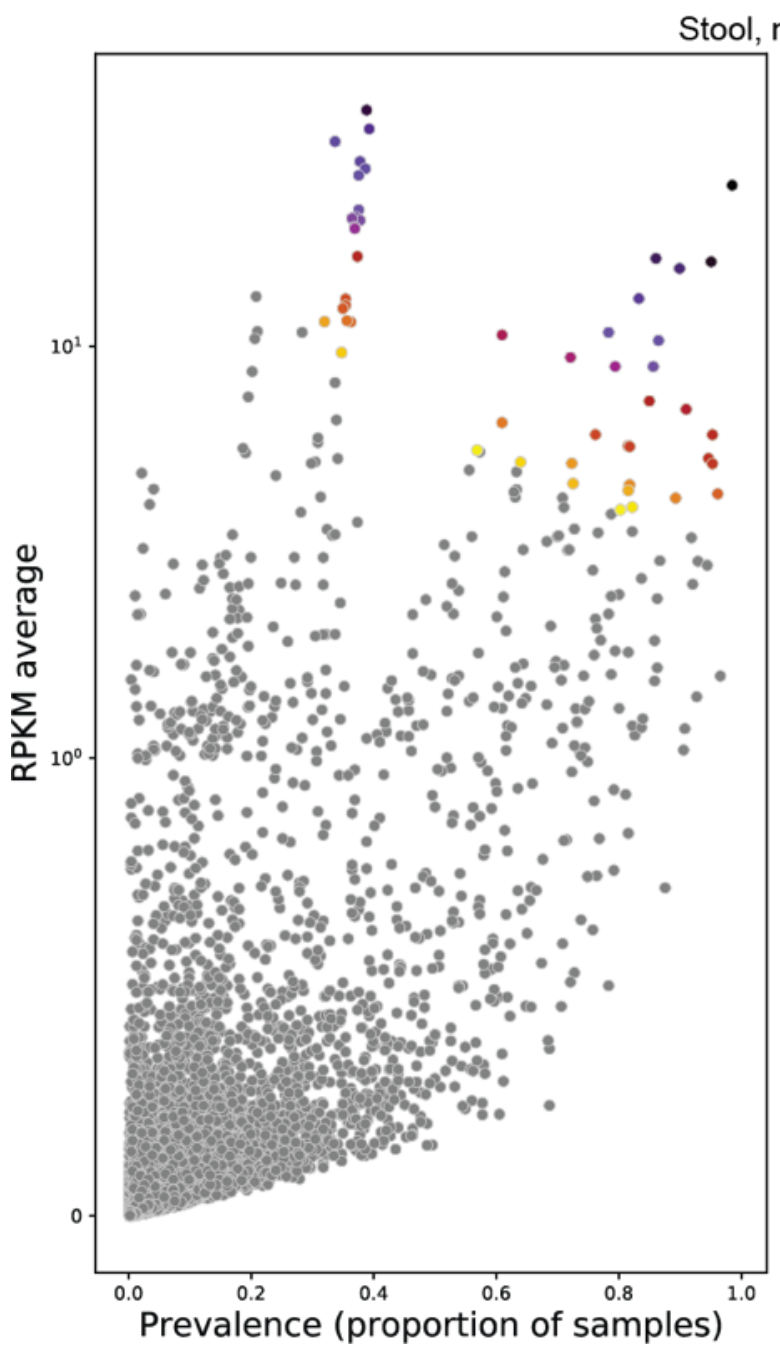

$$
n=466
$$

271 Figure 5: Most common viruses, Stool (Gut)

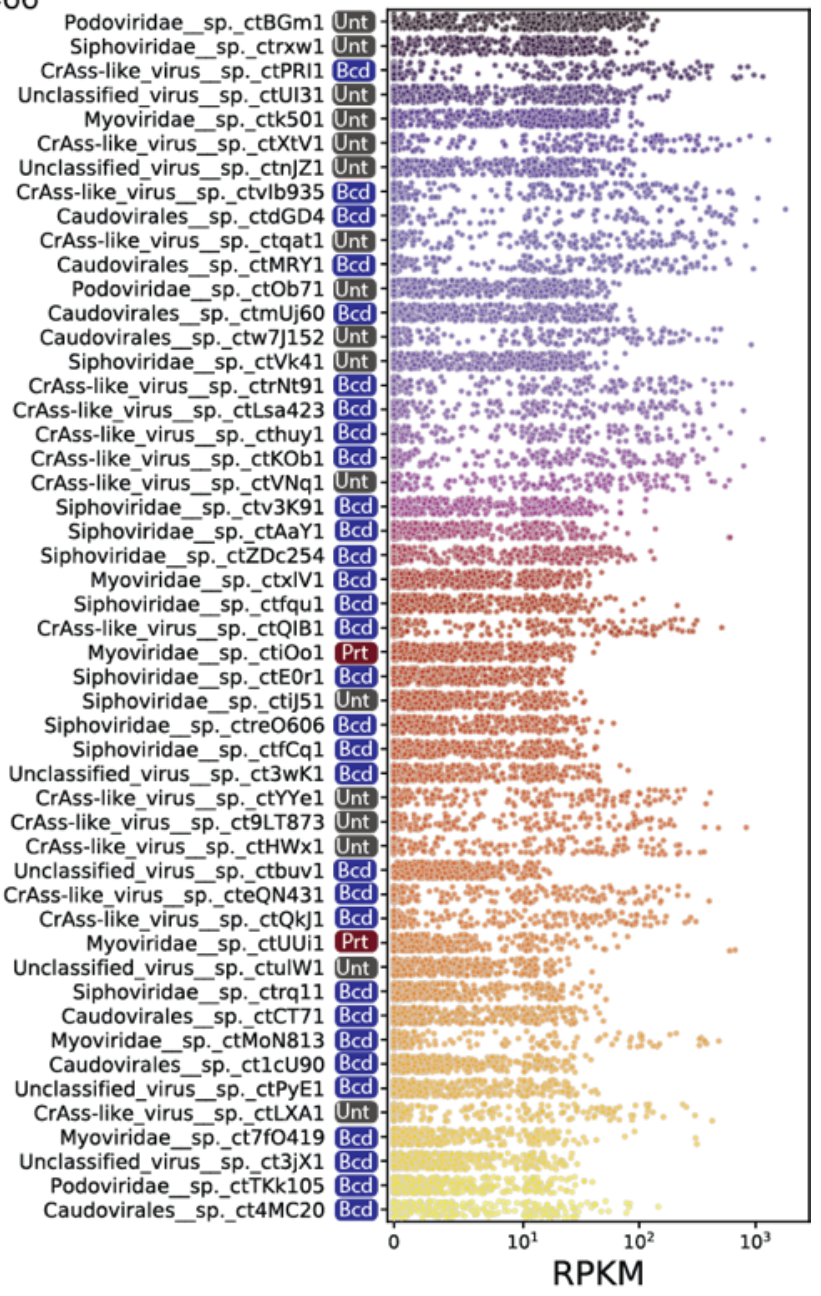

272 The left panel shows a scatter plot of RPKM (a measure of relative read abundance for a given

273 virus OTU, $y$-axis) versus prevalence (proportion of samples with >0.1 RPKM, $x$-axis). For display

274 purposes, the $y$-axis is a linear scale from $0-1\left(10^{\circ}\right)$ and $\log _{10}$ above 1 . The top fifty most

275 commonly abundant virus OTUs (based on the product of coordinates) are colored. Right panel:

276 RPKM values across all samples for the most commonly abundant virus OTUs. Colors of dots in

277 the left panel correspond with the dot strip colors in the right panel. The $x$-axis is linear from 0-

$27810\left(10^{1}\right)$ and $\log _{10}$ above 10. Colored CRISPR target logos: Bcd = Bacteroidetes, Prt =

279 Proteobacteria, Unt = no CRISPR spacers detected (untargeted). 
bioRxiv preprint doi: https://doi.org/10.1101/2020.11.01.363820; this version posted November 1,2020 . The copyright holder for this preprint (which was not certified by peer review) is the author/funder. This article is a US Government work. It is not subject to copyright under 17 USC 105 and is also made available for use under a CCO license.
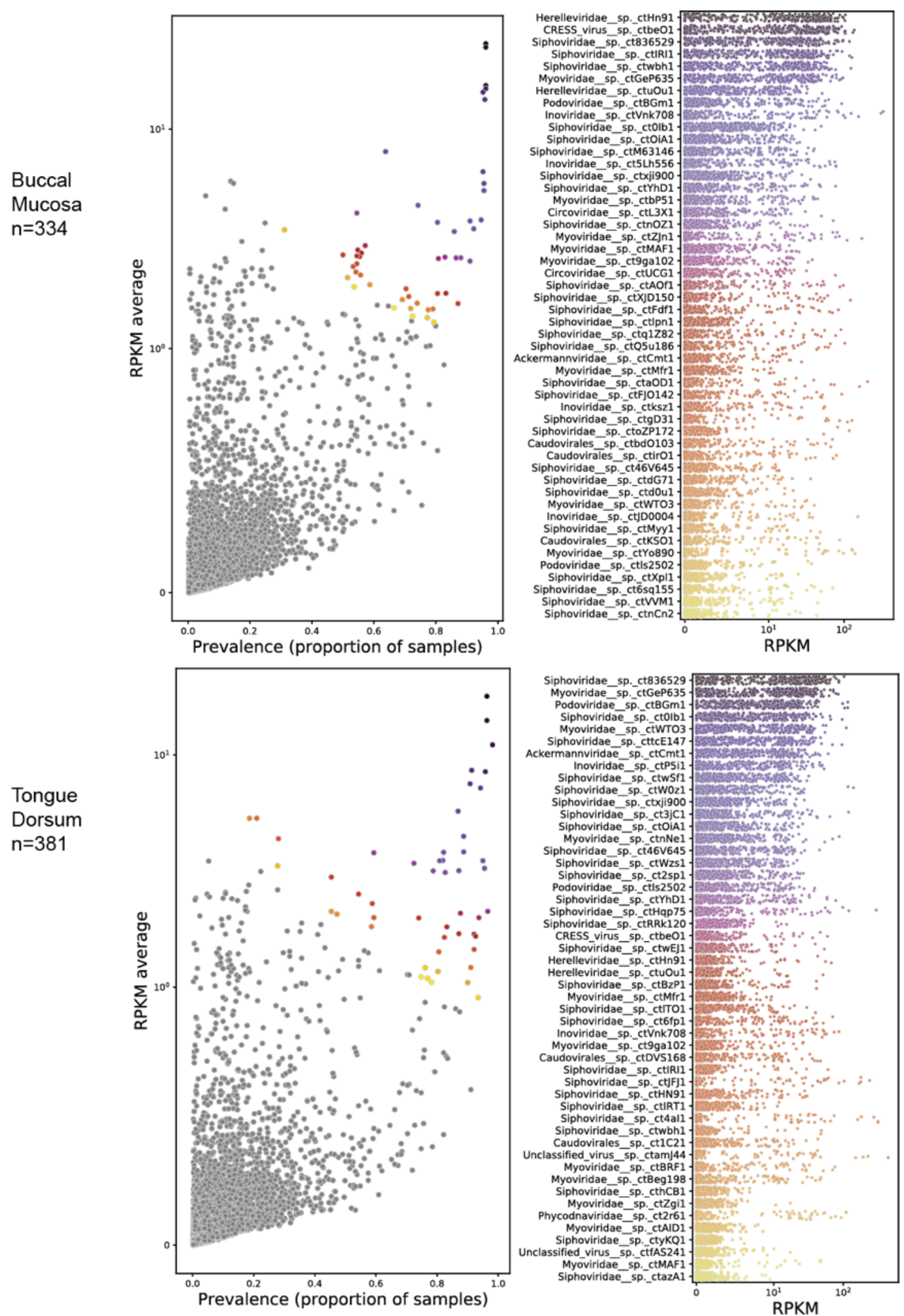

Supplemental Figure 2: Most common viruses, Buccal Mucosa and Tongue Dorsum 
bioRxiv preprint doi: https://doi.org/10.1101/2020.11.01.363820; this version posted November 1,2020. The copyright holder for this preprint (which was not certified by peer review) is the author/funder. This article is a US Government work. It is not subject to copyright under 17 USC 105 and is also made available for use under a CCO license.
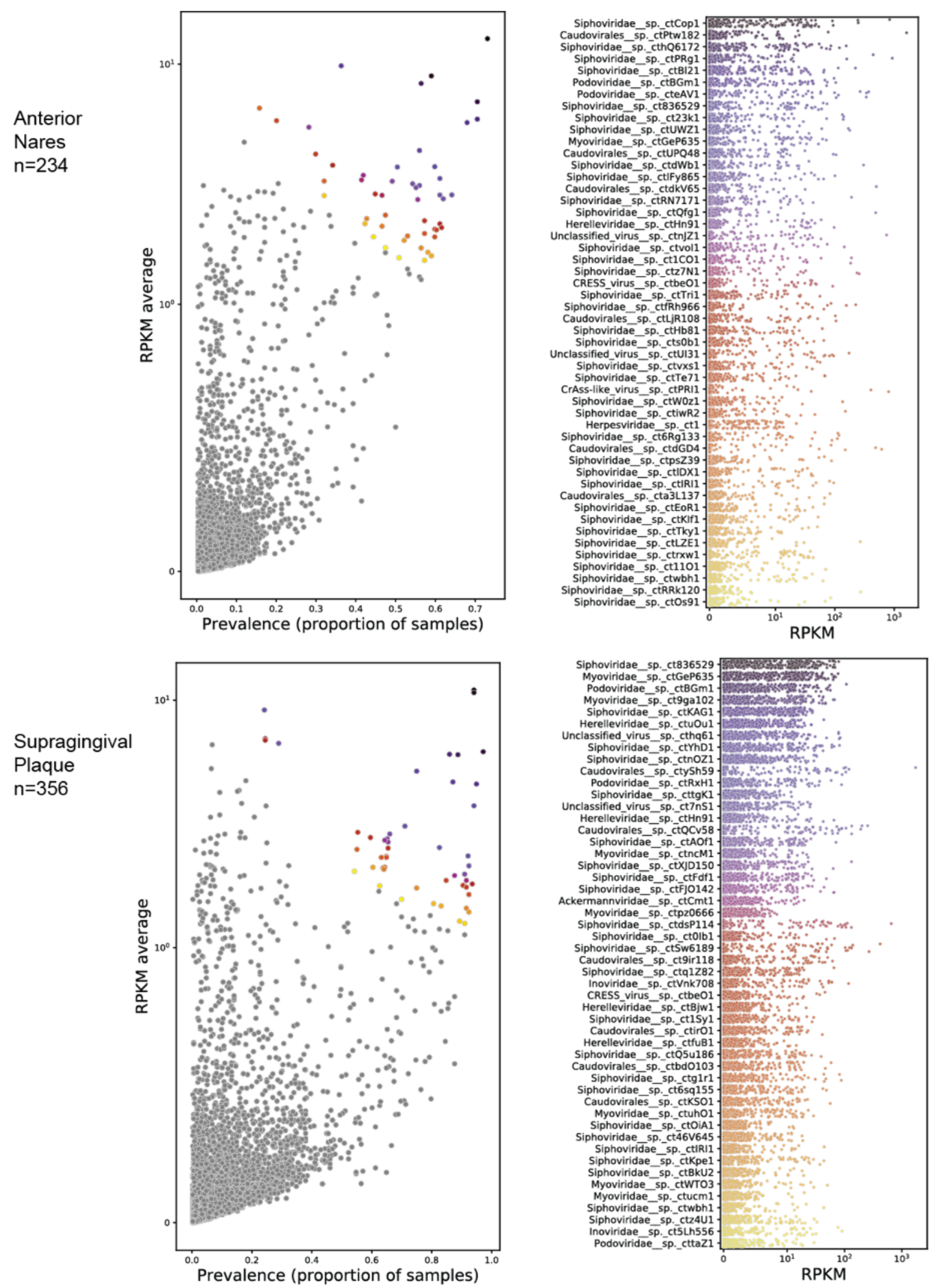

Supplemental Figure 3: Most common viruses, Anterior Nares and Supragingival Plaque 
bioRxiv preprint doi: https://doi.org/10.1101/2020.11.01.363820; this version posted November 1, 2020. The copyright holder for this preprint (which was not certified by peer review) is the author/funder. This article is a US Government work. It is not subject to copyright under 17 USC 105 and is also made available for use under a CCO license.
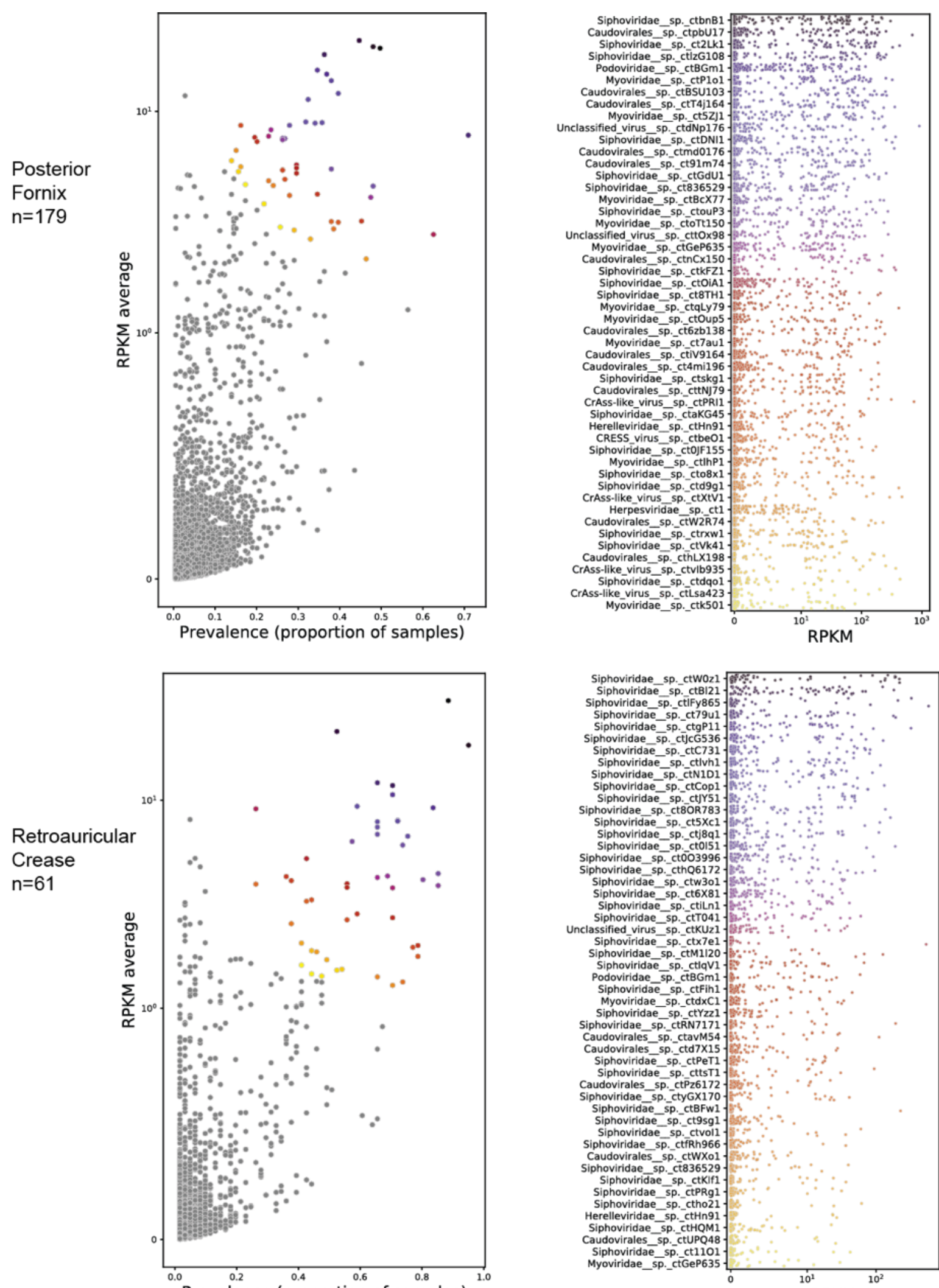

Prevalence (proportion of samples)

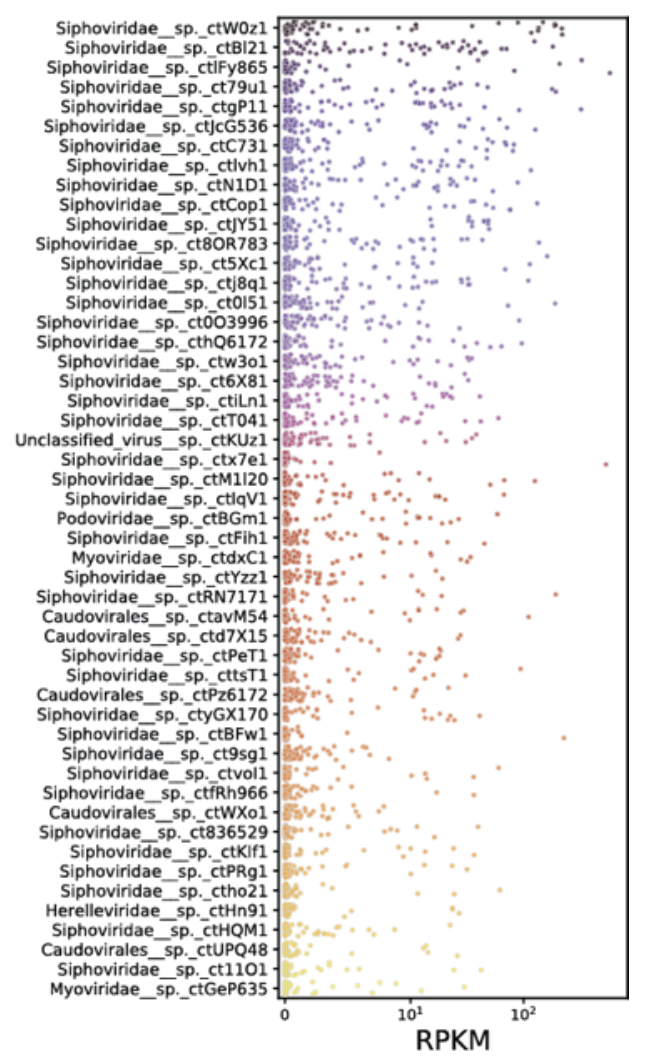

Supplemental Figure 4: Most common viruses, Posterior Fornix and Retroauricular Crease 
Specific virus OTUs are associated with human disease

A number of prior studies have looked for associations between the virome and human diseases $23-25,46,62-65$. However, these studies were limited by the lack of a comprehensive virus reference database, and nearly all studies examined samples selectively enriched for viral sequences $^{65}$. Virus enrichment methods can be highly variable and can inadvertently remove some viral taxa while failing to significantly select against host sequences ${ }^{9}{ }^{26}$. Indeed, Gregory et al. ${ }^{12}$ report that studies employing different virus-enrichment protocols to investigate the same disease state (e.g. inflammatory bowel disease) rarely contain the same virus populations in their data. Instead, studies using similar enrichment protocols (regardless of disease state of patients) shared more virus populations. Furthermore, sequences encapsidated within virions may not be the best reflection of the total viral population, especially in human digestive tracts, where many phages are believed to exist primarily in lysogenic (non-lytic) states ${ }^{66}$, and some have been 'grounded', losing their ability to independently excise from the host genome ${ }^{67}$. It is possible that the most important phages for human physiology are those that express accessory genes from an integrated provirus state, as opposed to phages that are producing virions. It is thus ideal to examine total DNA (also known as whole genome shotgun, WGS) sequencing, which can detect all DNA virus genomes.

Our study reanalyzed publicly available WGS data from twelve large case-control studies analyzing stool and/or saliva 29,35,68-74. These studies examined Parkinson's disease, obesity, colon carcinoma, colon adenoma, liver cirrhosis, type 1 diabetes, ankylosing spondylitis, atherosclerosis, type 2 diabetes, hypertension, and non-alcoholic fatty liver disease. The virus cores database was used to compare the abundance of each virus OTU between case and control cohorts. Figure 6 shows an analysis of case-control comparisons of Parkinson's disease (Fig. 6 A-C) and obesity (Fig. 6 D-F). RPKM was used to measure virus OTU abundance in each sample, and Wilcoxon rank-sum tests with 100 bootstraps were conducted for each comparison to estimate the $p$ value (Fig. 6A,D "Virome", Supplemental Figs. 5,6). Significant virus OTUs were determined by a false discovery rate $<1 \%$ (see Methods). All analyses compared associations between the virome and the "bacteriome," measuring the bacteriome in terms of bacterial OTUs (i.e. species-level single-copy bacterial marker gene abundance) using IGGsearch ${ }^{75}$ (Fig 6A,D "Bacteriome", Supplemental Figs. 5,6). A higher number of statistically significant taxa were found for the virome than the bacteriome in seven studies. The five other studies analyzed yielded no significant OTUs for either the virome or the bacteriome (Fig. 6, Supplemental Figs. 5,6). P values for each virus OTU detected in each study are documented in

323 Supplemental Table 2. Furthermore, random forest classifiers, trained on either all virus OTUs 324 or all bacterial OTUs, were more successful or equally successful, on average, in discriminating 325 healthy and diseased patients using the virome data rather than the bacteriome data in $7 / 12$ 326 case-control populations (Fig 6 B,E, Fig S7,8). 
bioRxiv preprint doi: https://doi. org/10.1101/202011.01.363820; this version posted November 1, 2020. The copyright holder for this preprint (which was not certified by peer review) is the author/funder. This article is a US Government work. It is not subject to copyright under 17 USC 105 and is also made available for use under a CCO license.

The importance of considering effect size when reporting microbiome associations has 328 become apparent in recent years ${ }^{76,77}$. Therefore, for all virus and bacterial OTUs with significant 329 differences between cases and controls, Cohen's d effect size is reported for each disease state 330 (Fig. 6C,F, Fig. S7,8). 
bioRxiv preprint doi: https://doi.org/10.1101/202011.01.363820; this version posted November 1, 2020. The copyright holder for this preprint (which was not certified by peer review) is the author/funder. This article is a US Government work. It is not subject to copyright under 17 USC 105 and is also made available for use under a CCO license.

A Parkinson's Disease, controls: $n=74$, Parkinson's disease: $n=108$
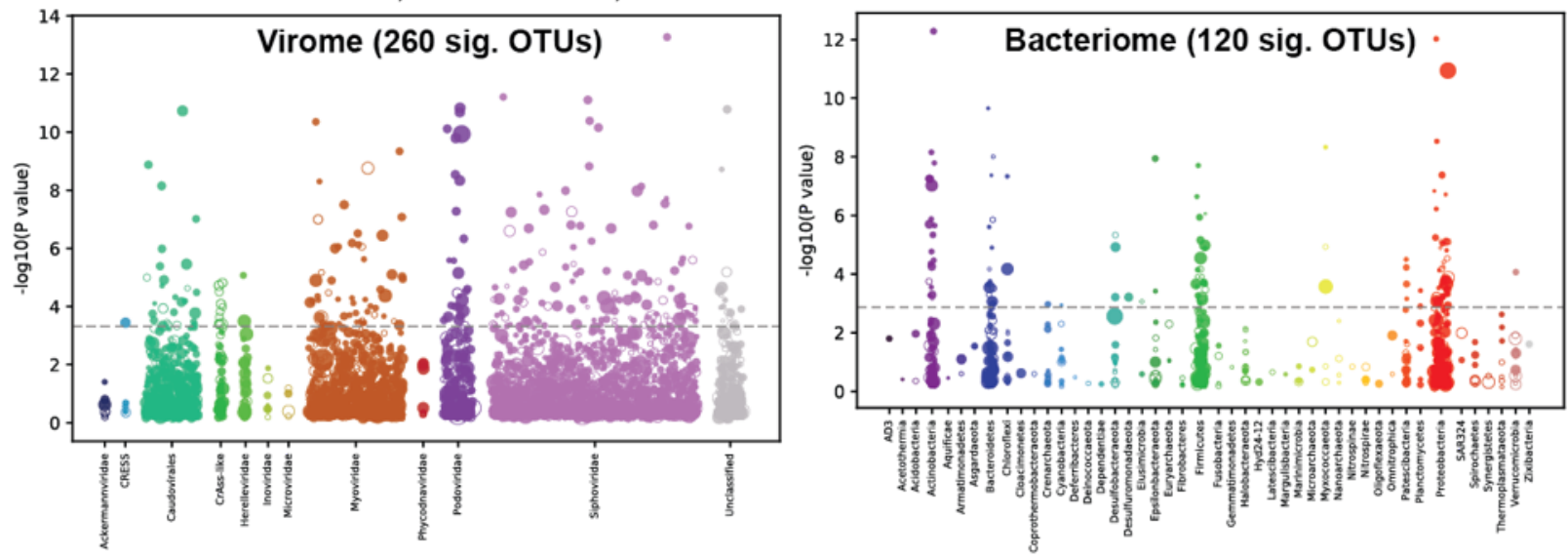

$\mathbf{B}$

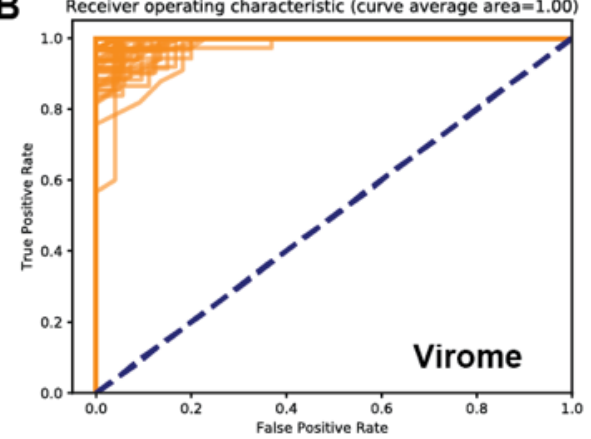

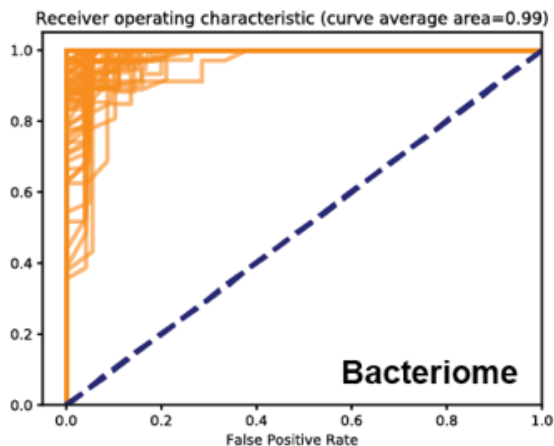

C Effect sizes of all significant taxa

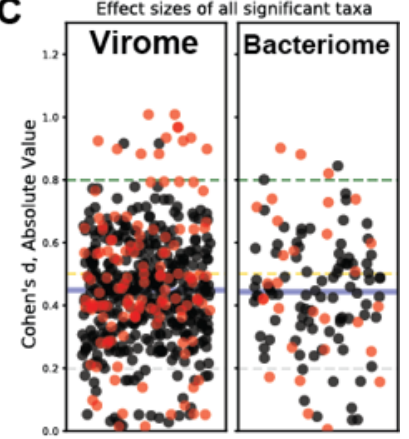

D Obesity, controls: $\mathrm{n}=235$, obese: $\mathrm{n}=360$
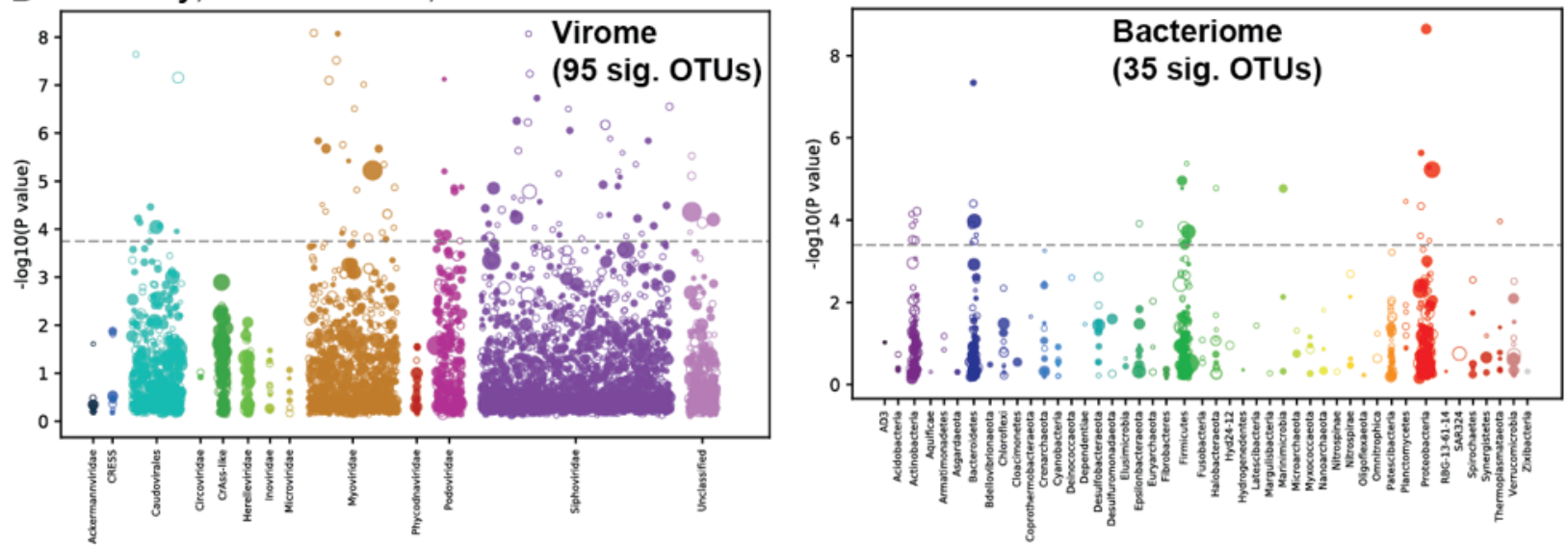

E
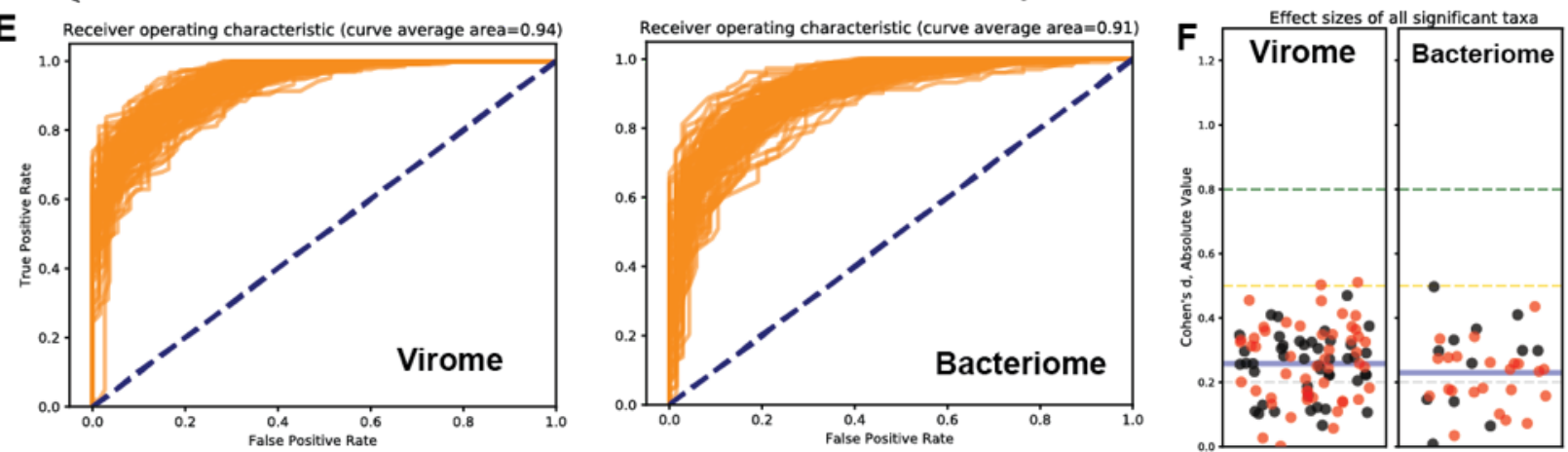

Figure 6: Association of the virome and bacteriome with chronic diseases 
333 (A-C) Analysis of read data from PRJEB17784, a case-control study of stool samples from

334 patients with or without Parkinson's disease. (A) Virome-wide and bacteriome-wide

335 associations in stool samples from Parkinson's disease patients $(n=74)$ and healthy controls

$336(n=108)$ represented as Manhattan plots. Each OTU is represented as a dot along the $X$-axis,

337 with its $y$-axis value being the inverse $\log _{10} p$ value. The size of each dot corresponds to the

338 median relative abundance of the taxon in the disease cohort. Filled dots represent OTUs found

339 at higher abundance in the diseased state while hollow dots represent decreased abundance in

340 the diseased sate. The dashed grey line represents the false discovery rate $<1 \%$ threshold. (B)

341 Receiver operating characteristic plots from 100 differently-seeded random forest classifiers

342 trained on the virome (left) or bacteriome (right). (C) Swarm plots of Cohen's d effect sizes

343 (absolute value) of OTUs achieving significant $p$ values. Black dots are positive effect size and

344 red dots are negative effect size. The mean of all plotted effect sizes is drawn as a blue line.

345 Small effect size $=0.2-0.5$; Medium effect size $=0.5-0.8$; Large effect size $=>0.8^{77}$. (D-F)

346 Similar analyses of read data from PRJEB4336, a WGS survey of stool samples from obese and

347 non-obese individuals. Plots $D, E$, and $F$ are laid out in the same manner as plots $A, B$, and C

348 respectively. 
bioRxiv preprint doi: https://doi.org/10.1101/2020.11.01.363820; this version posted November 1, 2020. The copyright holder for this preprint (which was not certified by peer review) is the author/funder. This article is a US Government work. It is not subject to copyright under 17 USC 105 and is also made available for use under a CCO license.

\section{Virome}

Colon adenoma (stool), controls: $n=126$, adenoma: $n=94$

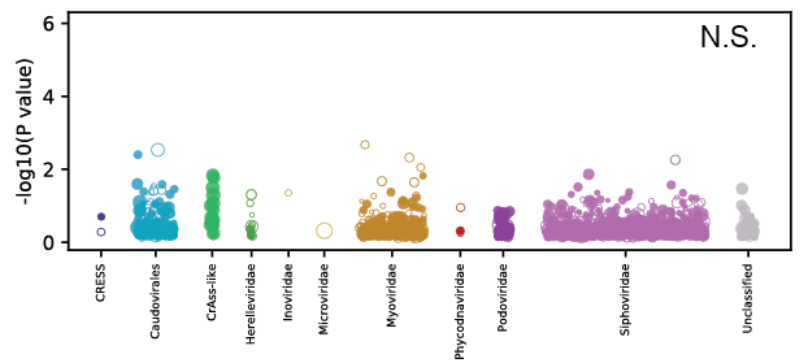

\section{Bacteriome}

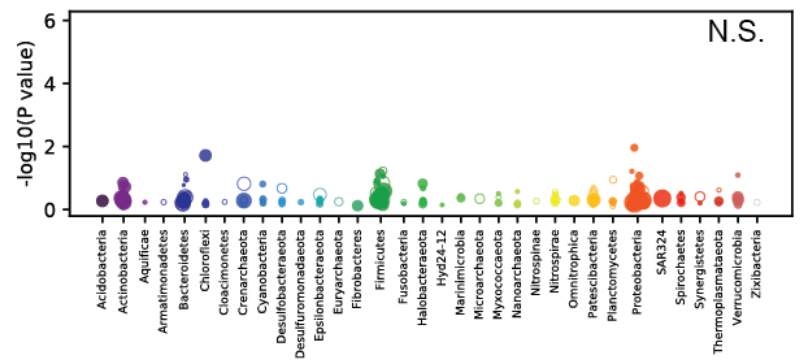

Colon carcinoma (stool), controls: $n=126$, carcinoma: $n=92$
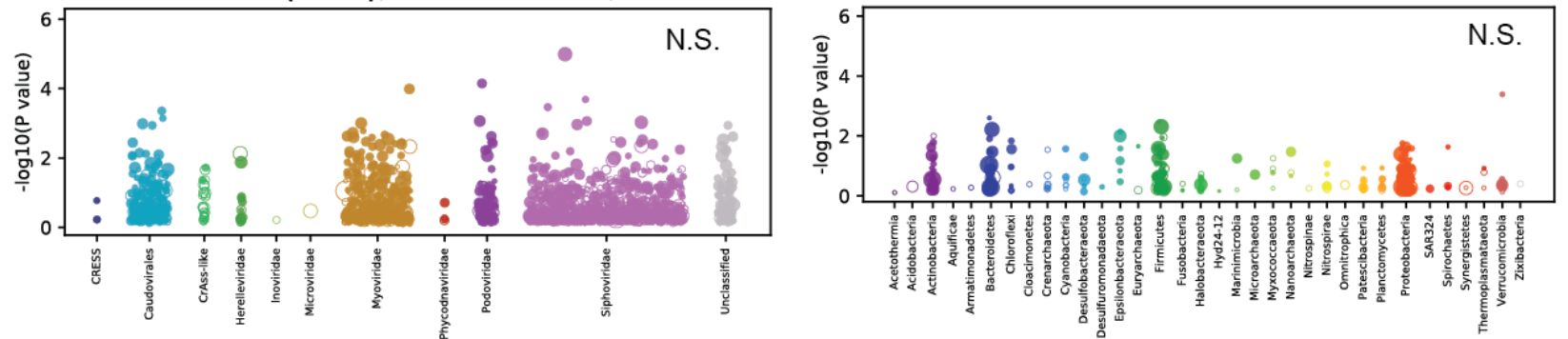

Liver cirrhosis (stool), controls: $n=145$, cirrhosis: $n=169$
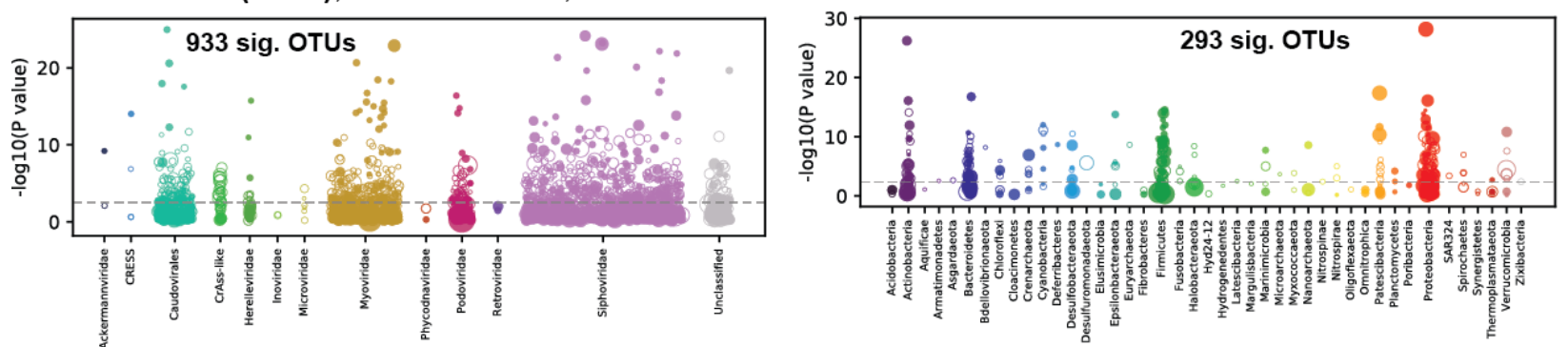

Type-1 Diabetes (stool), controls: $n=66$, diabetes: $n=87$
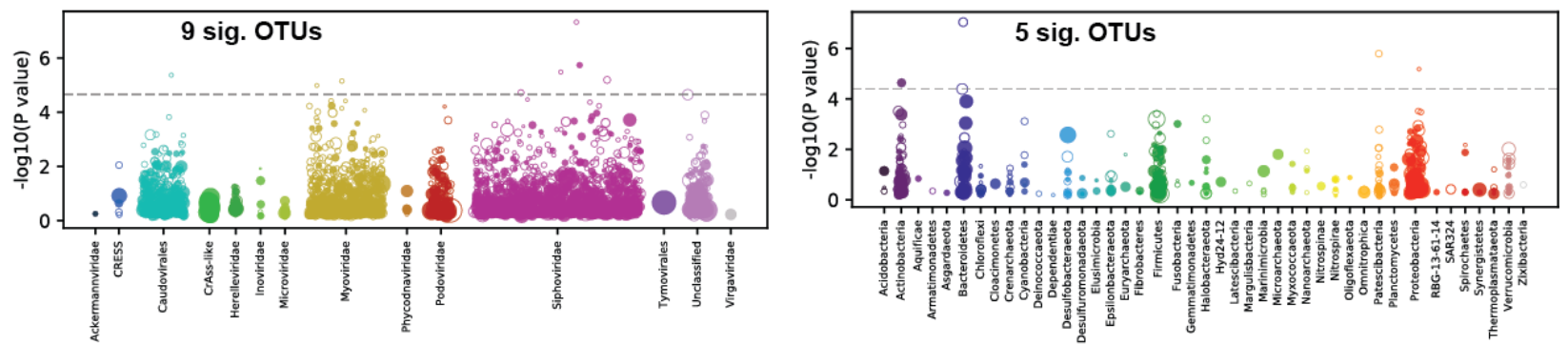

Type-1 Diabetes (saliva), controls: $n=40$, diabetes: $n=65$
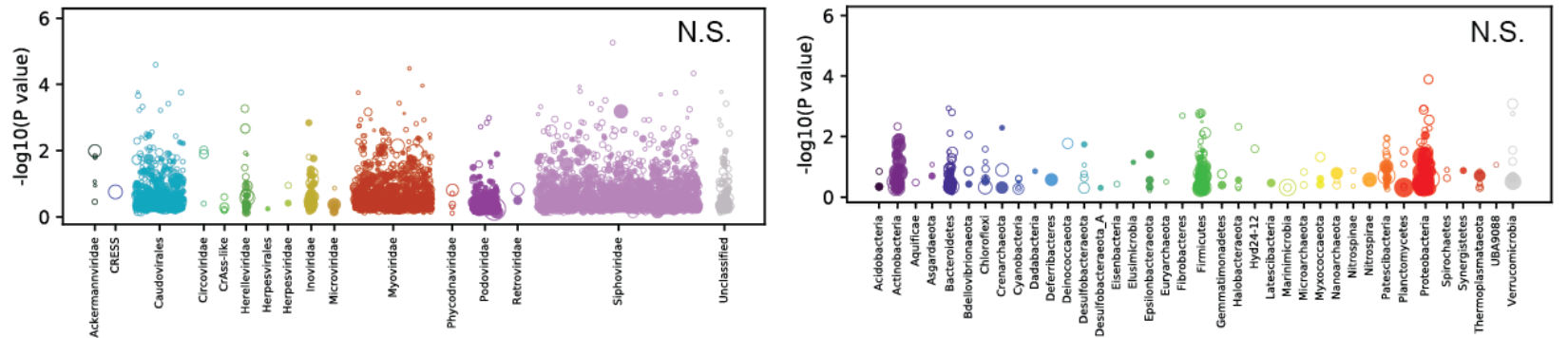

352 Supplemental Figure 5: Virome- and Bacteriome-wide associations with additional chronic 353 diseases 1 
bioRxiv preprint doi: https://doi.org/10.1101/202011.01363820 this version posted November 1, 2020. The copyright holder for this preprint (which was not certified by peer review) is the author/funder. This article is a US Government work. It is not subject to copyright under 17 USC 105 and is also made available for use under a CCO license.

354 Manhattan plots for viromes (left) and bacteriomes (right) are shown in the same manner as

355 Figure 6. Accessions: Colon carcinoma and adenoma (PRJEB7774), Liver cirrhosis (PRJEB6337), 356 Type 1 diabetes (PRJNA289586).

357 
bioRxiv preprint doi: https://doi.org/10.1101/2020.11.01.363820; this version posted November 1, 2020. The copyright holder for this preprint (which was not certified by peer review) is the author/funder. This article is a US Government work. It is not subject to copyright under 17 USC 105 and is also made available for use under a CCO license.

\section{Virome}

Ankylosing Spondylitis (stool), controls: $n=114$, ankylosing spondylitis: $n=97$

\section{Bacteriome}
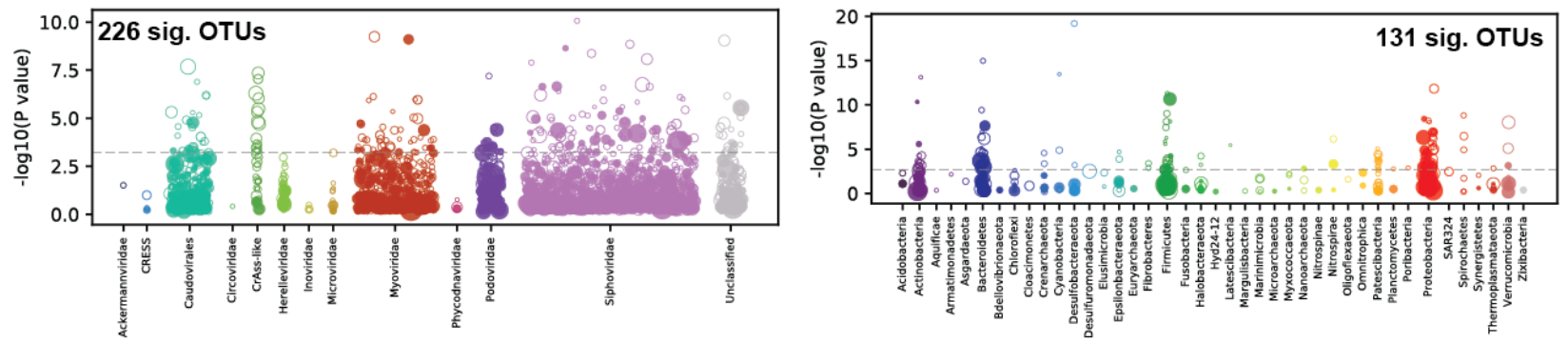

Type-2 Diabetes (stool), controls: $n=185$, type-2 diabetes: $n=177$
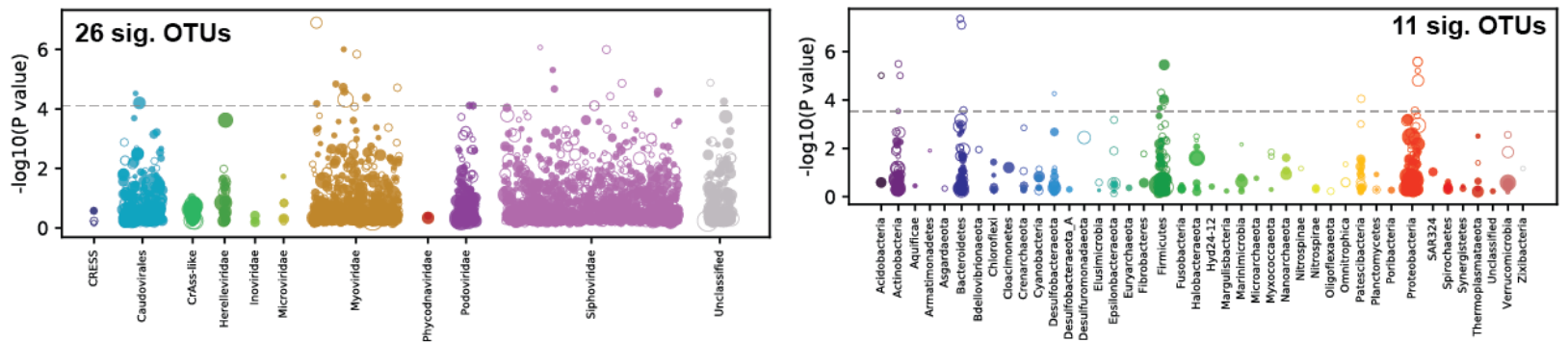

Atherosclerosis (stool), controls: $n=171$, atherosclertic: $n=176$
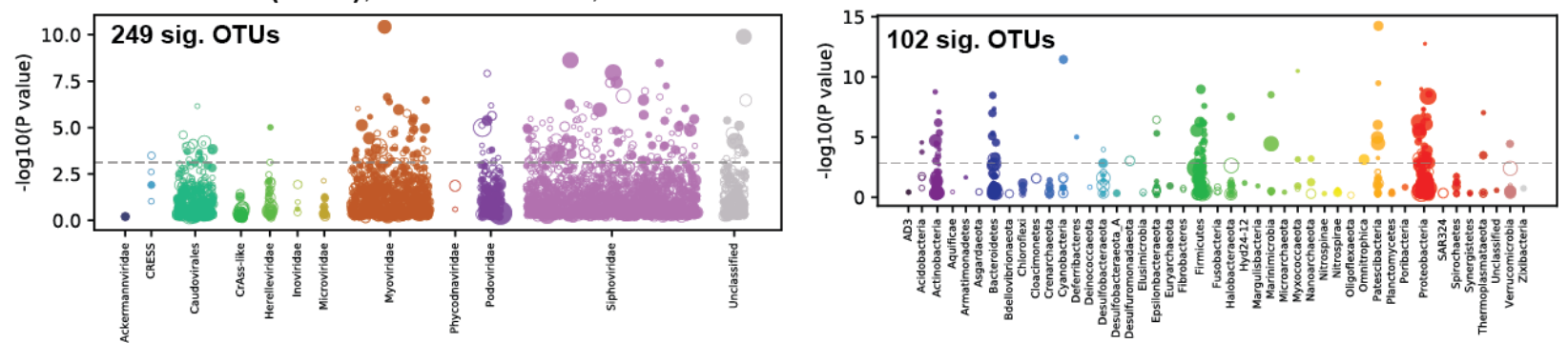

Hypertension (stool), controls: $n=41$, hypertensive: $n=99$
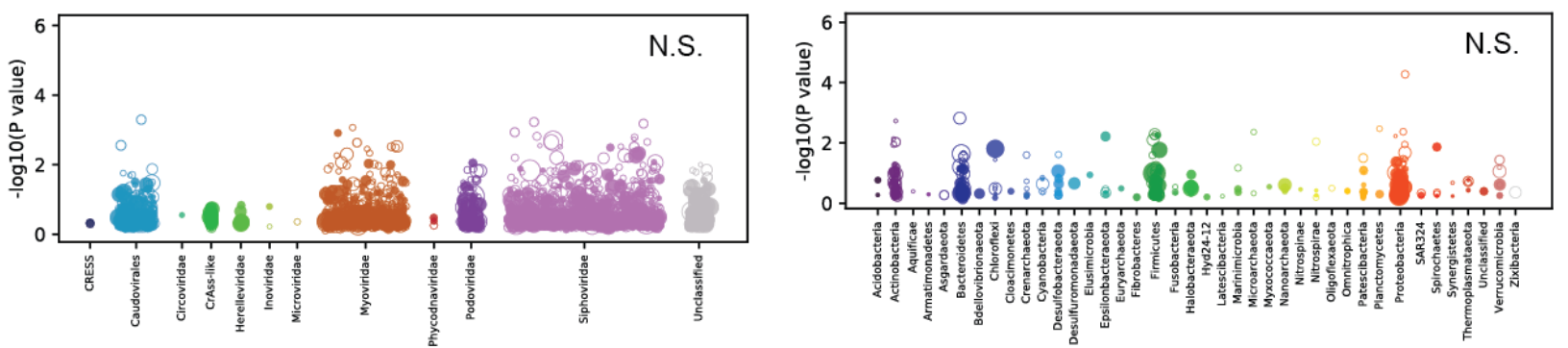

Fatty liver disease (stool), early stages $(0,1,2): n=72$, late stages $(3,4): n=14$
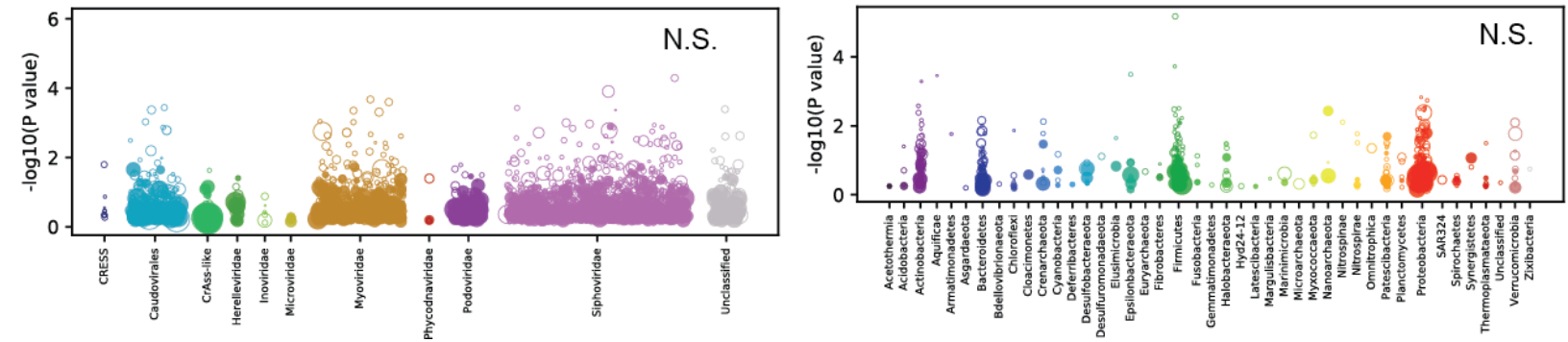

Supplemental Figure 6: Virome- and Bacteriome-wide associations with additional chronic diseases 2 
bioRxiv preprint doi: https://doi.org/10.1101/202011.01363820 this version posted November 1 2020. The copyright holder for this preprint (which was not certified by peer review) is the author/funder. This article is a US Government work. It is not subject to copyright under 17 USC 105 and is also made available for use under a CCO license.

361 Manhattan plots for stool viromes (left) and bacteriomes (right) are shown in the same manner 362 as Figure 6. Accessions: Ankylosing spondylitis (PRJNA375935), Type 2 diabetes (PRJNA422434), 363 Atherosclerosis (PRJEB21528), Hypertension (PRJEB13870), Fatty liver disease (PRJNA373901). 364 
bioRxiv preprint doi: https://doi.org/10.1101/202011.01363820 this version posted November 1, 2020. The copyright holder for this preprint (which was not certified by peer review) is the author/funder. This article is a US Government work. It is not subject to copyright under 17 USC 105 and is also made available for use under a CCO license.
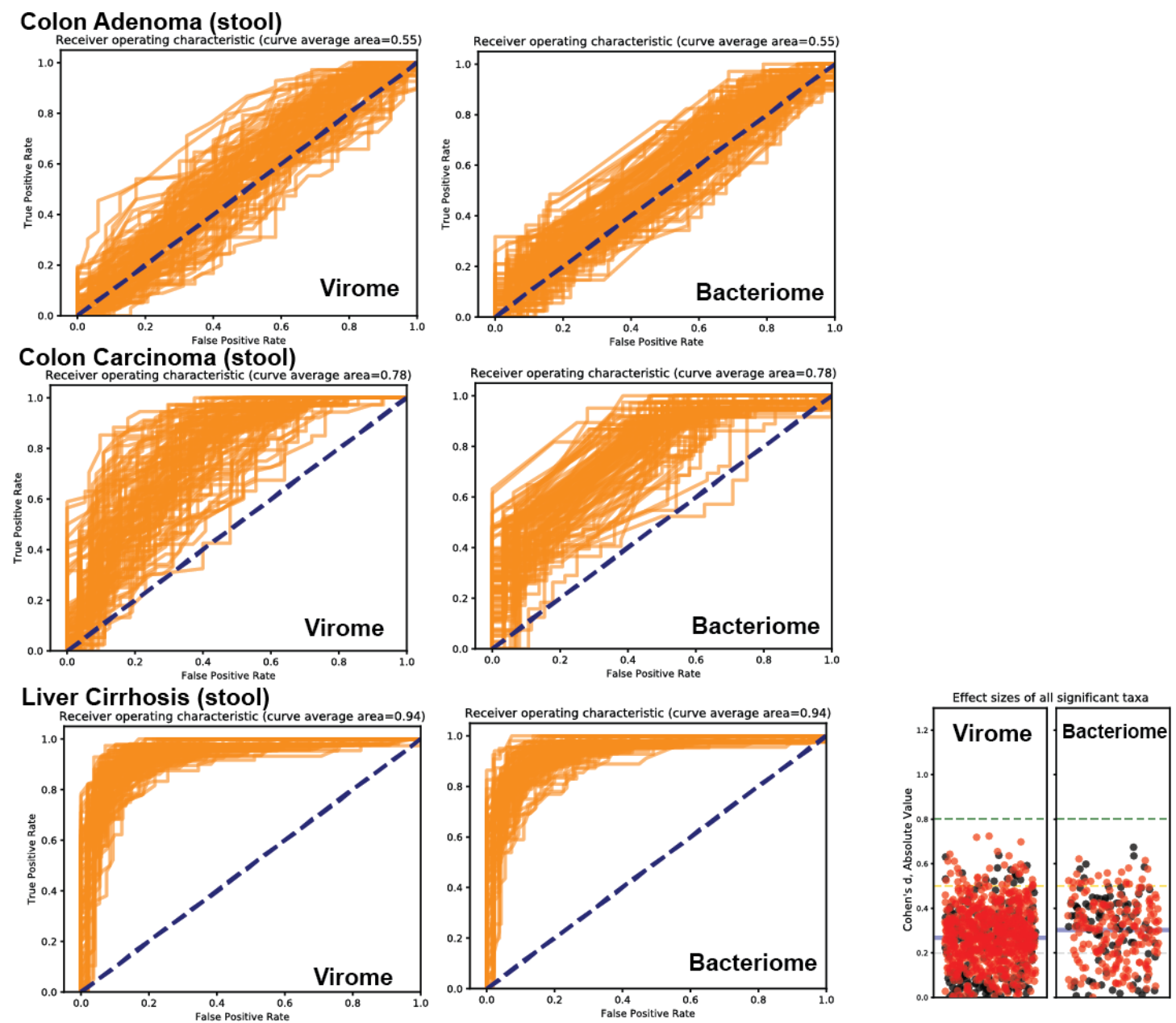

\section{Type-1 Diabetes (stool)}
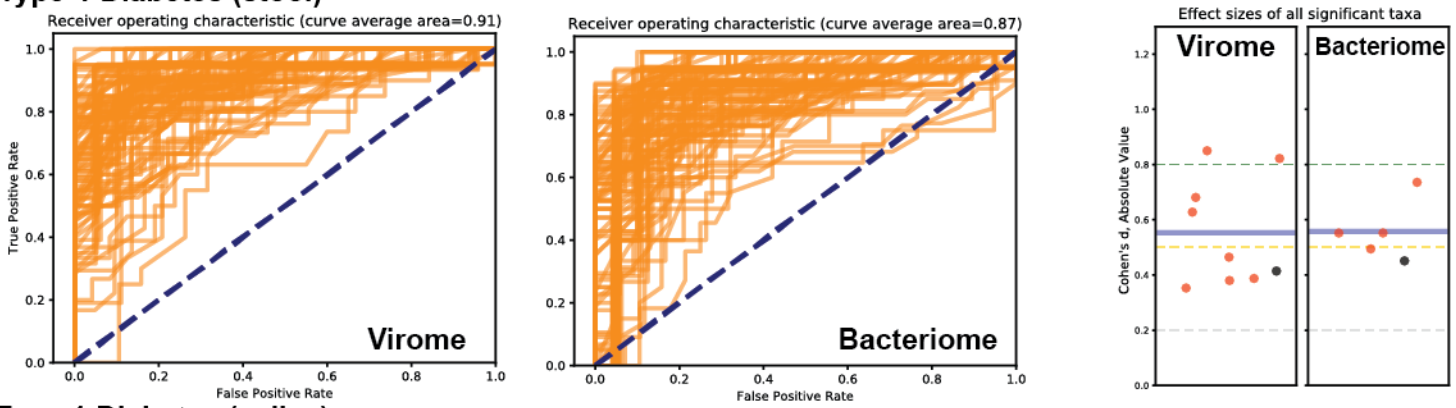

Type-1 Diabetes (saliva)
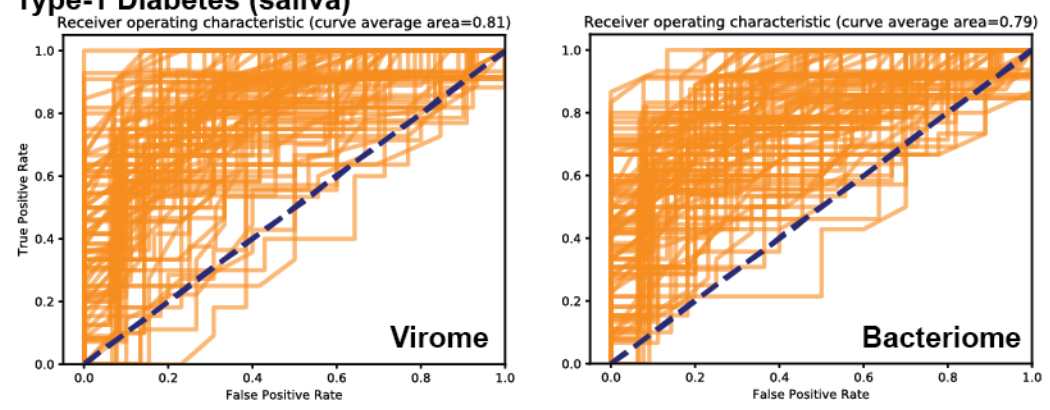

Supplemental Figure 7: Discriminatory power of viromes and bacteriomes in additional chronic 
bioRxiv preprint doi: https://doi.org/10.1101/202011.01363820 this version posted November 1, 2020. The copyright holder for this preprint

(which was not certified by peer review) is the author/funder. This article is a US Government work. It is not subject to copyright under 17 USC 105 and is also made available for use under a CCO license.

368 Receiver operating characteristic plots for virome (left panels) and bacteriome (middle panels) 369 data, as described in Figure 6. Summary of effect size data for significant OTUs (right panel), as 370 described in Figure 6.

371 
bioRxiv preprint doi: https://doi.org/10.1101/202011.01.363820; this version posted November 1, 2020. The copyright holder for this preprint (which was not certified by peer review) is the author/funder. This article is a US Government work. It is not subject to copyright under 17 USC 105 and is also made available for use under a CCO license.

Ankylosing Spondylitis (stool)

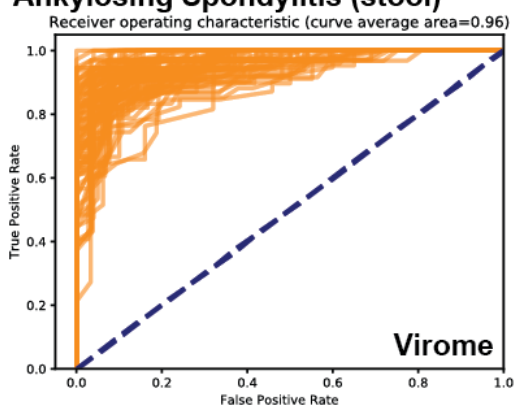

Type-2 Diabetes (stool)

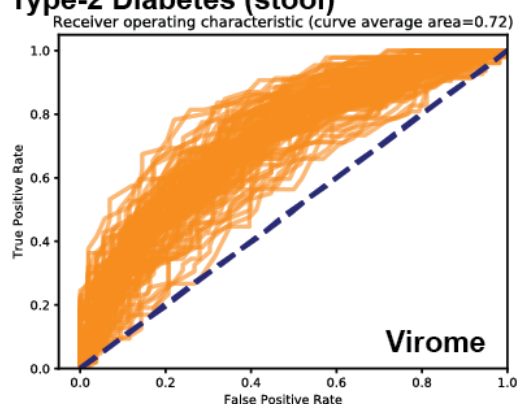

Atherosclerosis (stool)

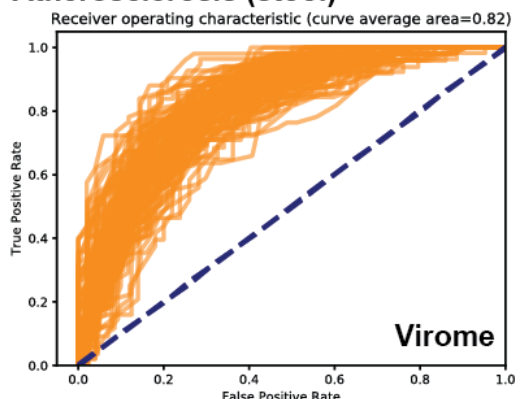

Hypertension (stool)

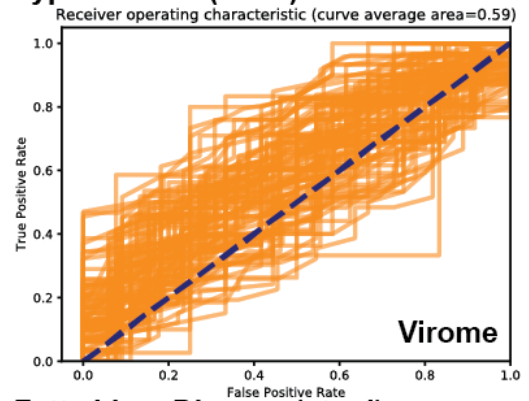

Fatty Liver Diseaase (stool)

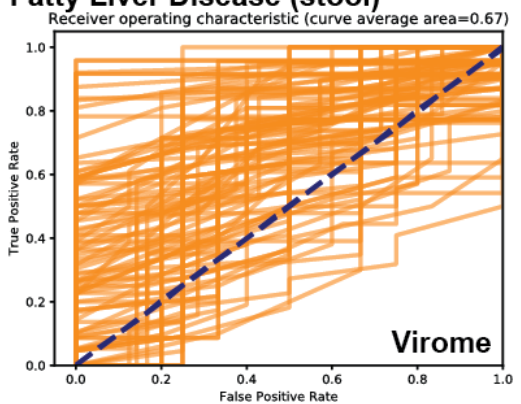

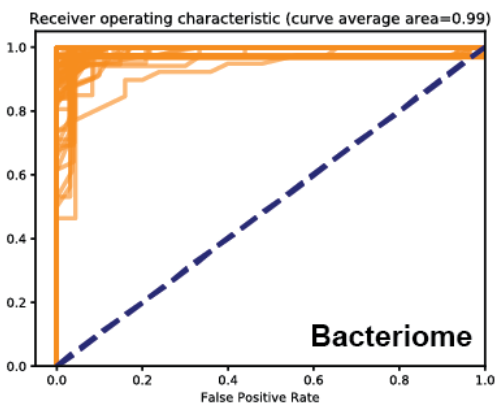
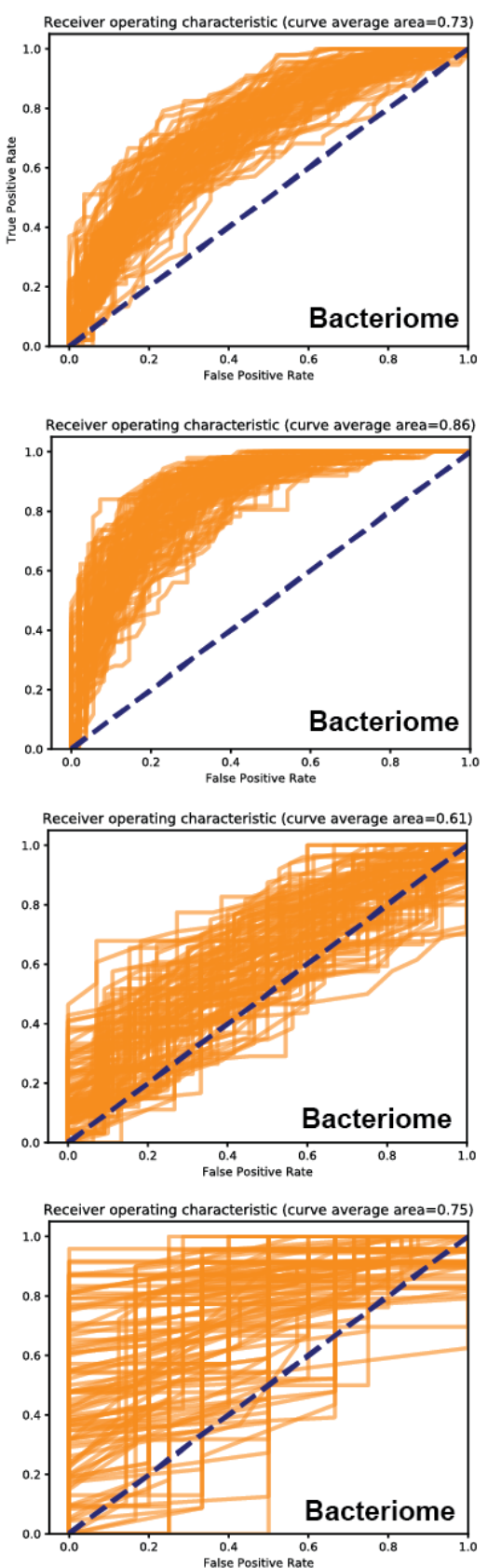
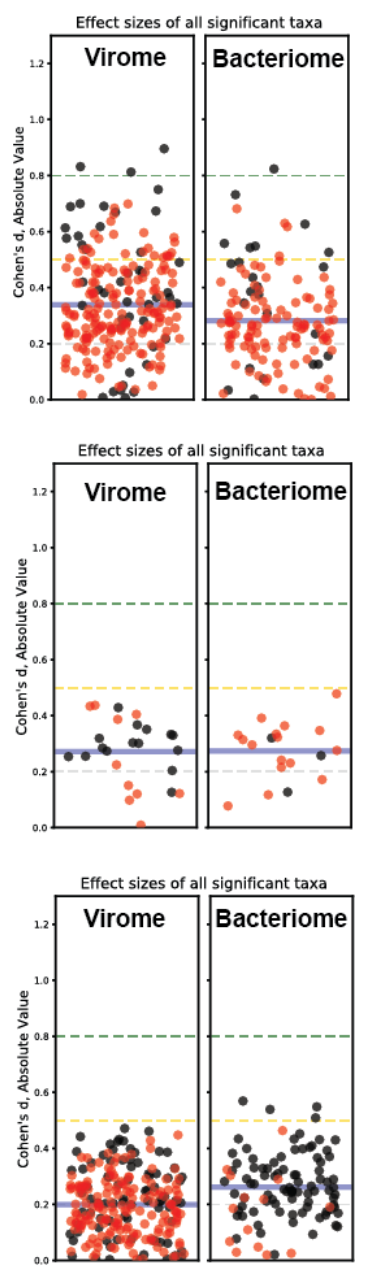
bioRxiv preprint doi: https://doi.org/10.1101/202011.01363820 this version posted November 1, 2020. The copyright holder for this preprint (which was not certified by peer review) is the author/funder. This article is a US Government work. It is not subject to copyright under 17 USC 105 and is also made available for use under a CCO license.

375 Receiver operating characteristic plots for virome (left panels) and bacteriome (middle panels) 376 data, as described in Figure 6. Summary of effect size data for significant OTUs (right panel), as 377 described in Figure 6. 


\section{Discussion}

This study shows that, by leveraging virus-specific hallmark genes, it is possible to mine human metagenomic data at a large scale to create a comprehensive database composed largely of previously unknown virus sequences. This advance, in turn, revealed hidden associations between a variety of chronic disease states and specific virus taxa. It should be stressed that association does not necessarily imply causation, and a variety of associative relationships between viruses and a given disease state are possible. For instance, virus abundance might simply be an epiphenomenon reflecting bacterial host abundance, the human genetics that predispose people to a disease might also provide a more favorable environment for the virus or its bacterial host, the external causes of a disease may create a more favorable environment for the virus, or the virus may contribute to the disease presentation in some way but ultimately does not cause the disease in isolation from other important factors. Verifying the associations we have detected with independent studies of the same diseases in additional populations will be key to understanding the extent to which the findings presented here are generalizable. If the associations are confirmed, it might be possible to experimentally test the causality question by adding or removing phages of interest from gut ecosystems in animal model systems ${ }^{78}$.

A limitation of the case-control studies analyzed here is that they only consisted of a single timepoint for each subject. Virome composition can be noisy, and longitudinal data on individual patients might be more effective for discerning stable viral populations ${ }^{10}$. This problem may have been partly offset by use of large cohort sizes (mostly over 150 total patients). Another limitation is that the analyzed case-control surveys only used DNA WGS methods whereas RNA sequencing of metatranscriptomes might have provided more functional data on expression of specific viral genes, potentially leading to testable hypotheses about possible mechanisms of action. It is also conceivable that correlations for viruses with RNA genomes would be uncovered. Despite these limitations, the current study shows that, using random forest classifiers, the virome may well be more diagnostic than the bacteriome for a variety of chronic diseases. The strong associations of specific virus OTUs in chronic diseases, along with medium to large effect sizes for many OTUs, cries out for more mechanistic investigation of possible causal roles for viruses in chronic human disease. 
415 Human Microbiome Project studies and other arbitrarily selected human metagenome studies

416 (Supplemental Table 1) were downloaded from SRA and unique Biosamples were delineated.

417 All runs from a given Biosample were downloaded concurrently, preprocessed with Fastp ${ }^{79}$, and

418 co-assembled with Megahit ${ }^{80}$ using default settings. Subsequent contigs were fed to Cenote-

419 Taker 2, with settings to consider circular or LTR-bearing contigs of at least $1500 \mathrm{nt}$, ITR-

420 containing contigs of at least $4 \mathrm{~kb}$, and linear contigs of at least $12 \mathrm{~kb}$. These contigs were

421 scanned for genes matching viral hallmark models and terminal repeat-containing contigs with

422 one or more viral hallmark genes were kept, as well as linear contigs with two or more viral

423 hallmark genes. Cenote-Taker 2 hallmark gene database was the September 15th, 2020 version

424 (see GitHub repo: https://github.com/mtisza1/Cenote-Taker2). While Cenote-Taker 2 does take

425 steps to earmark potential plasmids and conjugative transposons, extra precautions were taken

426 by removing $\sim 4000$ putative viral sequences from the non-redundant database that contained

427 replication-associated but not virion or genome-packaging hallmark genes. For

428 metatranscriptome datasets, all contigs over $1500 \mathrm{nt}$ with RNA virus hallmark genes were kept

429 as putative viral sequences, regardless of end features.

Clustering similar contigs

432 Mash $^{48}$ was used to cluster contigs into virus operational taxonomic units (OTUs) due to its

433 ability to handle massive sequence databases, accuracy, and lack of issues arising from genome

434 circularity. All viruses within each higher-level taxon (e.g. Microviridae) were used to create

435 Mash sketches (options -k 16 -s 500), and then these sketches were compared to themselves

436 with Mash's dist function. Within close genomic distances, the value of the Mash distance score

437 is thought to roughly recapitulate average nucleotide divergence. Virus strain-level distinctions

438 are often defined by $<5 \%$ average nucleotide divergence ${ }^{51}$, so sequence similarity networks

439 were constructed with connections between sequences (nodes) with Mash distance scores

$440 \leq 0.05$ (and $p$ value $\leq 1 \mathrm{e}^{-10}$ ). MCL clustering ${ }^{81}$ was applied to Mash networks to generate OTU-

441 level clusters. From each cluster of sequences, if circular or ITR-encoding sequences were

442 present, the longest such sequence was used as the representative virus OTU sequence. If only

443 linear sequences were present, the longest linear sequence was used as the representative of

444 the cluster. Singleton contigs (i.e., sequences that were not assigned to any cluster) were also

445 retained for the final database. The same approach was applied for the $99 \%$ database (for virus-

446 like particle sequence alignment), but a Mash distance score of $\leq 0.01$ was used.

448 Identifying cognate viruses in GenBank and the human Gut Virome Database

449 Using the NCBI Virus Resource, metadata for all virus genomes listed as complete were

450 downloaded for the following taxa: Adenoviridae, Anelloviridae, Bromoviridae, Caliciviridae,

451 Circoviridae, Cressdnavircota, Herpesviridae, Luteoviridae, Narnaviridae, Nodaviridae,

452 Papillomaviridae, Polyomaviridae, Tombusviridae, Totiviridae, Tymovirales, unclassified viruses, 
453 unclassified RNA virus, Virgaviridae, and all bacteriophage (including prophage). The metadata

454 were sorted so that the longest sequence for each unique species name was selected, and

455 these sequences were subsequently downloaded. Additionally, many GenBank virus genomes

456 simply have a family label followed by the indeterminate abbreviation "sp." and, as a result,

457 many highly distinct sequences inadvertently share an identical generic label. Therefore, all

458 complete GenBank virus genomes from all non-redundant taxa with an 'sp.' designation were

459 downloaded. A Mash sketch was made for the downloaded sequences using options (-k 16 -s

460 500), and this Mash sketch was compared to the Cenote Human Virome Database Mash sketch

461 (see above). Mash distances of $\leq 0.05$ and $p$ value $\leq 1 \mathrm{e}^{-10}$ were considered to be strict cognate

462 (intraspecies or intrastrain) sequences. Mash distances of $\leq 0.1$ and a $p$ value of $\leq 1 e^{-5}$ were used

463 for "loose" cognate sequences.

464 The Gut Virome Database from Gregory et al. ${ }^{12}$ was downloaded from: https://datacommons.cyverse.org/browse/iplant/home/shared/iVirus/Gregory_and_Zablocki_

466 GVD_Jul2020/GVD_Viral_Populations. The same Mash analyses were applied for comparisons

467 with this dataset as with the GenBank database.

Gene sharing network for unclassified viruses

470 Vcontact2 ${ }^{52,82}$ was run using all RefSeq v88 bacteriophage genomes with recommended

471 settings and all viruses from the Cenote Human Virome Database that were labeled

472 "unclassified" in the taxonomy field. The resulting network was displayed in Cytoscape ${ }^{83}$ and

473 colored manually.

474

Virus cores

476 Using all sequences from Cenote Human Virome Database (i.e. virus OTUs per 95\% clustering),

477 virus core coordinates were obtained. The Cenote-Taker 2 hallmark scan outputs for each

478 sequence were used to identify which genes encoded virus hallmarks (i.e. "hallmark genes").

479 The stop and start coordinates for each hallmark gene were compiled from the Cenote-Taker 2

480 output amino acid files. The coordinates with the lowest value and highest value were taken,

481 and bioawk was used to trim each fasta nucleotide sequence to start and end with these

482 coordinates, discarding outside sequences.

Bacteria-encoded CRISPR spacer analysis

485 CrisprOpenDB (https://github.com/edzuf/CrisprOpenDB, manuscript not currently available for 486 citation) was used (commit 04e4ffcc55d65cf8e13afe55e081b14773a6bb70) to assign phages to 487 hosts based on CRISPR spacer match. Three mismatches were allowed for hits. For hits to 488 bacteria without a currently assigned genus, family-level or order-level taxonomical information 489 was pulled from the output table, when possible. 


\section{Phage-encoded CRISPR spacer analysis}

All virus OTU sequences were processed with MinCED (https://github.com/ctSkennerton/minced) to discover CRISPR spacer arrays. As phages can encode CRISPR arrays with spacers as short as 14 nucleotides ${ }^{84}$, MinCED was allowed to detect arrays with spacers of 14 or more nucleotides. The CRISPR-array regions of phage genomes were masked using Bedtools maskfasta ${ }^{85}$, and then all virus OTUs were queried with BLASTN against a database of the CRISPR spacers.

Only hits aligning to the entire length of the spacer and with the following criteria were kept: perfect matches to spacers 16-20 nucleotides, matches to spacers 20-27 nucleotides where (mismatches + gaps) is 1 or 0 , matches to spacers $\geq 28$ nucleotides where (mismatches + gaps) is 2 or fewer.

Determining abundance of individual virus OTUs in metagenomes

504 The final database of "virus core" sequences was processed by RepeatMasker to remove low-

505 complexity regions which recruit reads non-specifically ${ }^{86}$. In the interest measuring known

506 human viruses that may have been missed by Cenote-Taker 2 analysis of human metagenomes, 410 viruses that reportedly infect humans were downloaded using the NCBI Virus Resource, processed by RepeatMasker, and added to the final database for read alignment (full-length sequences were kept for GenBank records). Bowtie $2^{87}$ was used to align reads to the database, and samtools ${ }^{88}$ idxstats was used to calculate read coverage and reads per kilobase per million reads (RPKM) for each contig.

Comparing OTU abundance and discriminatory ability in case-control studies

514 For each Bioproject, case vs control samples were determined, if possible, using supplemental 515 table 15 from Nayfach et al $^{75}$, as patients on confounding medications were removed in this 516 table. For other Bioprojects, metadata was taken from SRA ${ }^{89}$ run selector (Supplemental Table 517 6). For all samples, reads were downloaded from the SRA and trimmed and quality-controlled 518 with Fastp ${ }^{79}$. To quantify abundance of bacterial taxa in each sample, IGGsearch was used with 519 the "--all-species" option.

520 Wilcoxon rank-sum test was computed with 100 bootstraps using Python, NumPy and 521 SciPy $^{90}$ for each OTU in a given study where at least $10 \%$ of the total samples had an RPKM of at 522 least 0.05 (bacterial OTUs with "IGGsearch abundance" of at least 0.005 in at least $10 \%$ of the 523 samples were kept). False discovery rate (<1\%) was determined with the Benjamini-Hochberg 524 method using Scipy. Cohen's d effect size was calculated for each OTU above the significance 525 threshold using DaBest Python package ${ }^{91}$ with 5000 bootstraps.

$526 \quad$ Random Forest Classifiers from scikit-learn were used ${ }^{92}$. Training/test set sizes were 527 70\%/30\%, number of estimators was 100, and a different seed was used for each of the 100 528 Random Forest Classifiers trained on each dataset. 


\section{Data availability}

533 The fundamental data can be downloaded from: https://zenodo.org/record/4069377. At the

534 time of initial manuscript submission, deposition of each OTU in the CVHD into GenBank is in

535 process and accession numbers will be provided in a future revision.

536

537 Contributions

538 Michael J. Tisza: Conceptualization, Resources, Data curation, Formal analysis, Validation,

539 Investigation, Visualization, Methodology, Project administration, Writing manuscript

540 Christopher B. Buck: Conceptualization, Resources, Project administration, Editing manuscript

\section{Acknowledgements}

543 The authors would like to acknowledge Gabriel Starrett and Nathan Fons for their helpful

544 discussions on statistical analyses of the data types used in this paper. This work utilized the

545 computational resources of the NIH HPC Biowulf cluster. (http://hpc.nih.gov).

\section{Funding}

549 This research was funded by the NIH Intramural Research Program, with support from the $\mathrm{NCl}$ 550 Center for Cancer Research. 
5531 Pastrana, D. V. et al. Metagenomic Discovery of 83 New Human Papillomavirus Types in Patients with Immunodeficiency. mSphere 3, doi:10.1128/mSphereDirect.00645-18 (2018). 2 Beller, L. \& Matthijnssens, J. What is (not) known about the dynamics of the human gut
virome in health and disease. Curr Opin Virol 37, 52-57, doi:10.1016/j.coviro.2019.05.013 (2019). Gilbert, J. A. et al. Current understanding of the human microbiome. Nat Med 24, 392(2018)

5614 Manrique, P. et al. Healthy human gut phageome. Proc Natl Acad Sci U S A 113, 1040010405, doi:10.1073/pnas.1601060113 (2016). malnutrition. Proc Natl Acad Sci U S A 112, 11941-11946, doi:10.1073/pnas.1514285112 (2015). human feces. J Bacteriol 185, 6220-6223, doi:10.1128/jb.185.20.6220-6223.2003 (2003). Minot, S. et al. The human gut virome: inter-individual variation and dynamic response to diet. Genome Res 21, 1616-1625, doi:10.1101/gr.122705.111 (2011). Minot, S. et al. Rapid evolution of the human gut virome. Proc Natl Acad Sci U S A 110, 12450-12455, doi:10.1073/pnas.1300833110 (2013). faecal phageomes. Microbiome 6, 68, doi:10.1186/s40168-018-0446-z (2018). Shkoporov, A. N. et al. The Human Gut Virome Is Highly Diverse, Stable, and Individual Specific. Cell Host Microbe 26, 527-541 e525, doi:10.1016/j.chom.2019.09.009 (2019). Dutilh, B. E. et al. A highly abundant bacteriophage discovered in the unknown sequences of human faecal metagenomes. Nat Commun 5, 4498, doi:10.1038/ncomms5498 (2014).

12 Gregory, A. C. et al. The Gut Virome Database Reveals Age-Dependent Patterns of Virome Diversity in the Human Gut. Cell Host Microbe, doi:10.1016/j.chom.2020.08.003 (2020). patterns of adaptation across time and space. J Evol Biol 21, 1861-1866, doi:10.1111/j.1420-9101.2008.01598.x (2008).

14 Silveira, C. B. \& Rohwer, F. L. Piggyback-the-Winner in host-associated microbial communities. NPJ Biofilms Microbiomes 2, 16010, doi:10.1038/npjbiofilms.2016.10 (2016). host, bacteria and phages. Nat Rev Microbiol 15, 397-408, doi:10.1038/nrmicro.2017.30 (2017).

16 Gamage, S. D., Patton, A. K., Hanson, J. F. \& Weiss, A. A. Diversity and host range of Shiga toxin-encoding phage. Infect Immun 72, 7131-7139, doi:10.1128/IAI.72.12.71317139.2004 (2004). 
59417 Wagner, P. L. \& Waldor, M. K. Bacteriophage control of bacterial virulence. Infect Immun

595

596

597

598

599

600

601

602

603

604

605

606

607

608

609

610

611

612

613

614

615

616

617

618

619

620

621

622

623

624

625

626

627

$628 \quad 30$

629

630

631

632

633

634

635

636

70, 3985-3993, doi:10.1128/iai.70.8.3985-3993.2002 (2002).

18 Schuch, R. \& Fischetti, V. A. Detailed genomic analysis of the Wbeta and gamma phages infecting Bacillus anthracis: implications for evolution of environmental fitness and antibiotic resistance. J Bacteriol 188, 3037-3051, doi:10.1128/JB.188.8.3037-3051.2006 (2006).

19 Fridman, S. et al. A myovirus encoding both photosystem I and II proteins enhances cyclic electron flow in infected Prochlorococcus cells. Nat Microbiol 2, 1350-1357, doi:10.1038/s41564-017-0002-9 (2017).

20 Howard-Varona, C. et al. Phage-specific metabolic reprogramming of virocells. ISME J 14, 881-895, doi:10.1038/s41396-019-0580-z (2020).

21 Al-Shayeb, B. et al. Clades of huge phages from across Earth's ecosystems. Nature 578, 425-431, doi:10.1038/s41586-020-2007-4 (2020).

22 Tarafder, A. K. et al. Phage liquid crystalline droplets form occlusive sheaths that encapsulate and protect infectious rod-shaped bacteria. Proc Natl Acad Sci U S A 117, 4724-4731, doi:10.1073/pnas.1917726117 (2020).

23 Clooney, A. G. et al. Whole-Virome Analysis Sheds Light on Viral Dark Matter in Inflammatory Bowel Disease. Cell Host Microbe 26, 764-778 e765, doi:10.1016/j.chom.2019.10.009 (2019).

24 Norman, J. M. et al. Disease-specific alterations in the enteric virome in inflammatory bowel disease. Cell 160, 447-460, doi:10.1016/j.cell.2015.01.002 (2015).

25 Nakatsu, G. et al. Alterations in Enteric Virome Are Associated With Colorectal Cancer and Survival Outcomes. Gastroenterology 155, 529-541 e525, doi:10.1053/j.gastro.2018.04.018 (2018).

26 Zolfo, M. P., F.; Asnicar, F.; Manghi, P.; Tett, A.; Bushman, F. D.; Segata, N. Detecting contamination in viromes using ViromeQC. Nat Biotechnol 37, 1408-1412(2019), doi:https://doi.org/10.1038/s41587-019-0334-5 (2019).

27 Michael J. Tisza, A. K. B., Guillermo Dominguez-Huerta, Benjamin Bolduc, Matthew B. Sullivan, Christopher B. Buck. Cenote-Taker 2 Democratizes Virus Discovery and Sequence Annotation. BioRxiv, doi:https://doi.org/10.1101/2020.09.15.298943 (2020).

28 Lloyd-Price, J. et al. Strains, functions and dynamics in the expanded Human Microbiome Project. Nature 550, 61-66, doi:10.1038/nature23889 (2017).

$29 \mathrm{Li}$, J. et al. Gut microbiota dysbiosis contributes to the development of hypertension. Microbiome 5, 14, doi:10.1186/s40168-016-0222-x (2017).

30 Karlsson, F. H. et al. Gut metagenome in European women with normal, impaired and diabetic glucose control. Nature 498, 99-103, doi:10.1038/nature12198 (2013).

31 Backhed, F. et al. Dynamics and Stabilization of the Human Gut Microbiome during the First Year of Life. Cell Host Microbe 17, 852, doi:10.1016/j.chom.2015.05.012 (2015).

32 Horta-Baas, G. et al. Intestinal Dysbiosis and Rheumatoid Arthritis: A Link between Gut Microbiota and the Pathogenesis of Rheumatoid Arthritis. J Immunol Res 2017, 4835189, doi:10.1155/2017/4835189 (2017).

33 Pehrsson, E. C. et al. Interconnected microbiomes and resistomes in low-income human habitats. Nature 533, 212-216, doi:10.1038/nature17672 (2016). 
63734 Rampelli, S. et al. Metagenome Sequencing of the Hadza Hunter-Gatherer Gut

638 Microbiota. Curr Biol 25, 1682-1693, doi:10.1016/j.cub.2015.04.055 (2015).

63935 Heintz-Buschart, A. et al. Integrated multi-omics of the human gut microbiome in a case 640 study of familial type 1 diabetes. Nat Microbiol 2, 16180,

641

64236 Karlsson, F., Tremaroli, V., Nielsen, J. \& Backhed, F. Assessing the human gut microbiota in metabolic diseases. Diabetes 62, 3341-3349, doi:10.2337/db13-0844 (2013).

37 Olm, M. R. et al. Identical bacterial populations colonize premature infant gut, skin, and oral microbiomes and exhibit different in situ growth rates. Genome Res 27, 601-612, doi:10.1101/gr.213256.116 (2017). metagenomic analysis. Sci Rep 6, 34826, doi:10.1038/srep34826 (2016).

39 Qin, J. et al. A metagenome-wide association study of gut microbiota in type 2 diabetes. Nature 490, 55-60, doi:10.1038/nature11450 (2012). metagenome. Nature 514, 59-64, doi:10.1038/nature13786 (2014). 150,000 Genomes from Metagenomes Spanning Age, Geography, and Lifestyle. Cell 176, 649-662 e620, doi:10.1016/j.cell.2019.01.001 (2019).

Koenig, J. E. et al. Succession of microbial consortia in the developing infant gut microbiome. Proc Natl Acad Sci U S A 108 Suppl 1, 4578-4585, doi:10.1073/pnas.1000081107 (2011). Maqsood, R. et al. Discordant transmission of bacteria and viruses from mothers to babies at birth. Microbiome 7, 156, doi:10.1186/s40168-019-0766-7 (2019). Yinda, C. K. et al. Gut Virome Analysis of Cameroonians Reveals High Diversity of Enteric Viruses, Including Potential Interspecies Transmitted Viruses. mSphere 4, doi:10.1128/mSphere.00585-18 (2019). Coffey, M. J. et al. The intestinal virome in children with cystic fibrosis differs from healthy controls. PLoS One 15, e0233557, doi:10.1371/journal.pone.0233557 (2020). Zuo, T. et al. Gut mucosal virome alterations in ulcerative colitis. Gut 68, 1169-1179, doi:10.1136/gutjnl-2018-318131 (2019). cohort of adult men. Nat Microbiol 3, 356-366, doi:10.1038/s41564-017-0084-4 (2018).

48 Ondov, B. D. et al. Mash: fast genome and metagenome distance estimation using MinHash. Genome Biol 17, 132, doi:10.1186/s13059-016-0997-x (2016).

49 Beaulaurier, J. et al. Assembly-free single-molecule sequencing recovers complete virus genomes from natural microbial communities. Genome Res 30, 437-446, doi:10.1101/gr.251686.119 (2020).

50 Koonin, E. V. et al. Global Organization and Proposed Megataxonomy of the Virus World. Microbiol Mol Biol Rev 84, doi:10.1128/MMBR.00061-19 (2020).

51 Roux, S. et al. Minimum Information about an Uncultivated Virus Genome (MIUViG). Nat Biotechnol 37, 29-37, doi:10.1038/nbt.4306 (2019). 
67952 Bin Jang, H. et al. Taxonomic assignment of uncultivated prokaryotic virus genomes is

680 enabled by gene-sharing networks. Nat Biotechnol 37, 632-639, doi:10.1038/s41587-

681 019-0100-8 (2019).

$68253 \quad$ Gregory, A. C. et al. Marine DNA Viral Macro- and Microdiversity from Pole to Pole. Cell 177, 1109-1123 e1114, doi:10.1016/j.cell.2019.03.040 (2019).

685

54 Tisza, M. J. et al. Discovery of several thousand highly diverse circular DNA viruses. Elife 9, doi:10.7554/eLife.51971 (2020).

687

55 Roux, S. et al. Cryptic inoviruses revealed as pervasive in bacteria and archaea across Earth's biomes. Nat Microbiol 4, 1895-1906, doi:10.1038/s41564-019-0510-x (2019). marine phages. Front Microbiol 5, 27, doi:10.3389/fmicb.2014.00027 (2014). Makarova, K. S. et al. Evolution and classification of the CRISPR-Cas systems. Nat Rev Microbiol 9, 467-477, doi:10.1038/nrmicro2577 (2011). Briner, A. E. et al. Occurrence and Diversity of CRISPR-Cas Systems in the Genus Bifidobacterium. PLoS One 10, e0133661, doi:10.1371/journal.pone.0133661 (2015).

59 Seed, K. D., Lazinski, D. W., Calderwood, S. B. \& Camilli, A. A bacteriophage encodes its own CRISPR/Cas adaptive response to evade host innate immunity. Nature 494, 489491, doi:10.1038/nature11927 (2013). Faure, G. et al. CRISPR-Cas in mobile genetic elements: counter-defence and beyond. Nat Rev Microbiol 17, 513-525, doi:10.1038/s41579-019-0204-7 (2019). doi:10.1038/nature06244 (2007). Zhao, G. et al. Intestinal virome changes precede autoimmunity in type I diabetessusceptible children. Proc Natl Acad Sci U S A 114, E6166-E6175, doi:10.1073/pnas.1706359114 (2017). Ulcerative Colitis and Crohn Disease. J Pediatr Gastroenterol Nutr 68, 30-36, doi:10.1097/MPG.0000000000002140 (2019). Kramna, L. et al. Gut virome sequencing in children with early islet autoimmunity. Diabetes Care 38, 930-933, doi:10.2337/dc14-2490 (2015). Ma, Y., You, X., Mai, G., Tokuyasu, T. \& Liu, C. A human gut phage catalog correlates the gut phageome with type 2 diabetes. Microbiome 6, 24, doi:10.1186/s40168-018-0410-y (2018). Front Endocrinol (Lausanne) 10, 784, doi:10.3389/fendo.2019.00784 (2019).

67 Ramisetty, B. C. M. \& Sudhakari, P. A. Bacterial 'Grounded' Prophages: Hotspots for Genetic Renovation and Innovation. Front Genet 10, 65, doi:10.3389/fgene. 2019.00065 (2019).

68 Le Chatelier, E. et al. Richness of human gut microbiome correlates with metabolic markers. Nature 500, 541-546, doi:10.1038/nature12506 (2013).

69 Loomba, R. et al. Gut Microbiome-Based Metagenomic Signature for Non-invasive Detection of Advanced Fibrosis in Human Nonalcoholic Fatty Liver Disease. Cell Metab 30, 607, doi:10.1016/j.cmet.2019.08.002 (2019). 
72270 Wen, C. et al. Quantitative metagenomics reveals unique gut microbiome biomarkers in

723 ankylosing spondylitis. Genome Biol 18, 142, doi:10.1186/s13059-017-1271-6 (2017).

72471 Bedarf, J. R. et al. Functional implications of microbial and viral gut metagenome

725

726

727 changes in early stage L-DOPA-naive Parkinson's disease patients. Genome Med 9, 39, doi:10.1186/s13073-017-0428-y (2017).

728

72 Qin, N. et al. Alterations of the human gut microbiome in liver cirrhosis. Nature 513, 5964, doi:10.1038/nature13568 (2014).

730

Jie, Z. et al. The gut microbiome in atherosclerotic cardiovascular disease. Nat Commun

732

74 Zhang, X. et al. The oral and gut microbiomes are perturbed in rheumatoid arthritis and partly normalized after treatment. Nat Med 21, 895-905, doi:10.1038/nm.3914 (2015). uncultivated genomes of the global human gut microbiome. Nature 568, 505-510, doi:10.1038/s41586-019-1058-x (2019).

76 Debelius, J. et al. Tiny microbes, enormous impacts: what matters in gut microbiome studies? Genome Biology 17, doi:ARTN 217

738 10.1186/s13059-016-1086-x (2016).

73977 Sawilowsky, S. S. New Effect Size Rules of Thumb. J Mod App/ Stat Meth 8, 597-599, doi:DOI 10.22237/jmasm/1257035100 (2009). with Challenges. Cell Host Microbe 19, 575-578, doi:10.1016/j.chom.2016.04.014 (2016).

79 Chen, S. F., Zhou, Y. Q., Chen, Y. R. \& Gu, J. fastp: an ultra-fast all-in-one FASTQ preprocessor. Bioinformatics 34, 884-890, doi:10.1093/bioinformatics/bty560 (2018).

81 Morris, J. H. et al. clusterMaker: a multi-algorithm clustering plugin for Cytoscape. BMC

82 Devisetty, U. K., Kennedy, K., Sarando, P., Merchant, N. \& Lyons, E. Bringing your tools to CyVerse Discovery Environment using Docker. F1000Res 5, 1442, doi:10.12688/f1000research.8935.1 (2016). Cytoscape 3. Curr Protoc Bioinformatics 47, 8.13.11-24, doi:10.1002/0471250953.bi0813s47 (2014).

764 Pausch, P. et al. CRISPR-CasPhi from huge phages is a hypercompact genome editor. Science 369, 333-337, doi:10.1126/science.abb1400 (2020).

85 Quinlan, A. R. BEDTools: The Swiss-Army Tool for Genome Feature Analysis. Curr Protoc Bioinformatics 47, 1112 11-34, doi:10.1002/0471250953.bi1112s47 (2014).

86 Chen, N. Using RepeatMasker to identify repetitive elements in genomic sequences. Curr Protoc Bioinformatics Chapter 4, Unit 4 10, doi:10.1002/0471250953.bi0410s05 (2004).

87 Langdon, W. B. Performance of genetic programming optimised Bowtie2 on genome comparison and analytic testing (GCAT) benchmarks. BioData Min 8, 1, doi:10.1186/s13040-014-0034-0 (2015). 
76688 Li, H. et al. The Sequence Alignment/Map format and SAMtools. Bioinformatics 25,

767 2078-2079, doi:10.1093/bioinformatics/btp352 (2009).

76889 Kodama, Y., Shumway, M., Leinonen, R. \& International Nucleotide Sequence Database, 769 C. The Sequence Read Archive: explosive growth of sequencing data. Nucleic Acids Res

770 40, D54-56, doi:10.1093/nar/gkr854 (2012).

$77190 \quad$ Virtanen, P. et al. SciPy 1.0: fundamental algorithms for scientific computing in Python.

$772 \quad$ Nat Methods 17, 261-272, doi:10.1038/s41592-019-0686-2 (2020).

77391 Ho, J., Tumkaya, T., Aryal, S., Choi, H. \& Claridge-Chang, A. Moving beyond P values: data

774 analysis with estimation graphics. Nat Methods 16, 565-566, doi:10.1038/s41592-019-

775 0470-3 (2019).

77692 Abraham, A. et al. Machine learning for neuroimaging with scikit-learn. Front

$777 \quad$ Neuroinform 8, 14, doi:10.3389/fninf.2014.00014 (2014). 